\title{
Adaptive Organizations
}

\section{Wouter Dessein}

University of Chicago

Tano Santos

Columbia University and National Bureau of Economic Research

\begin{abstract}
We consider organizations that optimally choose the level of adaptation to a changing environment when coordination among specialized tasks is a concern. Adaptive organizations provide employees with flexibility to tailor their tasks to local information. Coordination is maintained by limiting specialization and improving communication. Alternatively, by letting employees stick to some preagreed action plan, organizations can ensure coordination without communication, regardless of the extent of specialization. Among other things, our theory shows how extensive specialization results in organizations that ignore local knowledge, and it explains why improvements in communication technology may reduce specialization by pushing organizations to become more adaptive.
\end{abstract}

\section{Introduction}

Since Adam Smith ([1776] 1981), specialization and the division of labor have been at the center of the organization of production. Whereas Smith emphasized how the division of labor is limited by the "extent of the market," many economists have argued that, perhaps more important, the benefits of specialization are limited by the need to coor-

We thank Gary Becker, Patrick Bolton, Mathias Dewatripont, Jonathan Levin, Kevin Murphy, Canice Prendergast, John Roberts, and participants in numerous workshops for helpful comments. A previous version of this paper was circulated under the title "The Demand for Coordination." All errors remain our own.

[ Journal of Political Economy, 2006, vol. 114, no. 5]

(C) 2006 by The University of Chicago. All rights reserved. 0022-3808/2006/11405-0005 $\$ 10.00$ 
dinate specialized activities. ${ }^{1}$ A common implication of these theories is that observed improvements in communication and information technology in recent decades should generate more specialization in the workplace. Indeed, information technology increases the size of the market by eliminating boundaries of space and time, and it allows for a better coordination of specialized activities. This prediction, however, is seemingly at odds with a growing body of empirical and anecdotal evidence that has documented a trend toward "new workplace practices" involving less specialized job assignments, more teamwork, and more intensive communication. ${ }^{2}$ In this paper we propose a new theory of organizational design that can account for this trend.

Our starting point is that a potential goal of organizations is to adapt to a changing environment but that the information needed to do so may be dispersed among employees; that is, information is local in nature. Organizations optimally choose how "adaptive" and thus how "information intensive" to be, by which we mean how much to make use of this local information. In particular, organizations can provide employees with the flexibility to tailor their actions to the local information they alone observe. Alternatively, they can let employees stick to some preagreed course of action and ignore local information. This affects how coordination is achieved. In the former case, when organizations are adaptive, coordination between specialized employees must be achieved ex post, using costly and imperfect communication. Here the organization may reduce coordination costs by limiting specialization. In contrast, in the latter organization, no communication is required since employee behavior is perfectly predictable. Coordination is then achieved ex ante and is unaffected by the extent of specialization. Thus, in our setup, specialization is limited by how adaptive or information intensive the organization is.

The paper endogenizes the organization's choice of adaptiveness and therefore how the organization should be structured in terms of how many tasks to give to employees and how much to promote communication between them. This theory has implications for organizational

\footnotetext{
${ }^{1}$ See, e.g., Becker and Murphy (1992), Bolton and Dewatripont (1994), and Garicano (2000). Also, the business literature has identified and paid considerable attention to this trade-off. Indeed, according to Rivkin and Siggelkow (2003, 292), "the [qualitative management] literature is unified in what it perceives as the central challenge of organizational design: to divide the tasks of a firm into manageable, specialized jobs, yet coordinate the tasks so that the firm reaps the benefits of harmonious action." For an early reference on the statement of the problem, see March and Simon (1958, 22-30).

${ }^{2}$ See, e.g., Osterman (1994), Ichniowski et al. (1996), Brynjolfsson and Hitt (1998), OECD (1999), Caroli (2001), and Caroli and Van Reenen (2001). Consistent with this trend toward less specialized jobs, the biggest management fad of the 1990s, reengineering (Hammer and Champy 2001, 70) prescribes "combining several jobs into one" and thus "putting back together again the work that Adam Smith and Henry Ford broke into tiny pieces."
} 
design very different from those so far considered in the literature. For example, improvements in communication technology favor information-intensive organizations and may therefore result in less specialization, not more. As already mentioned, this accords better with the observed trends in organizational design.

We propose a simple team-theoretic model of an organization that must perform $n$ tasks. The overall organizational success depends on how well each of these tasks is adapted to its own idiosyncratic needs and how well all tasks are coordinated with each other. In particular, each task consists of a primary action, which should be set as close as possible to some "local information," as well as $n-1$ complementary actions, which should be set as close as possible to the primary actions of the other $n-1$ tasks. Organizational design consists in determining (i) how many tasks an employee carries out and (ii) how much an employee can tailor his primary action to his local information. As has been stressed ever since Adam Smith, we assume that there are positive returns to having fewer tasks per employee. The problem is that only the employee in charge of a particular task observes the local information pertaining to that task. Hence, specialized employees must communicate their private information to each other in order to ensure effective coordination, and such communication is imperfect. While we initially treat the quality of communication as an exogenous parameter, we also analyze communication as an endogenous organizational design variable. That is, organizations can foster communication between specialized employees at a cost. This reflects the opportunity cost of having employees engaged in regular meetings, conference calls, and electronic mail conversations, designed to exchange information, rather than in production itself.

A number of insights emerge from the analysis. First, organizations reduce the division of labor as the business environment becomes more uncertain. The organization then optimally allows employees more flexibility in carrying out their task, increasing the need for ex post coordination through task bundling. Conversely, as the Smithian returns to specialization increase, organizations become more rigid and provide their employees with less flexibility to adapt to local circumstances.

Second, specialization is nonmonotonic in improvements in communication technology. On the one hand, better or cheaper communication reduces the need to coordinate through task bundling (Bolton and Dewatripont 1994; Garicano 2000). On the other hand, organizations take advantage of better or cheaper communication to become more adaptive, increasing the need for task bundling. For example, when communication is limited or very costly, organizations may optimally force agents to stick to some preagreed course of action, in which case task bundling has no value. But improvements in communication 
technology may transform such a rigid, specialized organization into a flexible one characterized by broad task assignments and substantial employee flexibility. This is consistent with a body of evidence that shows that information technology and the adoption of new work practicesemphasizing employee autonomy and broad job definitions-cluster together (see, e.g., Brynjolffson and Hitt 1998; Bresnahan, Brynjolffson, and Hitt 2002).

Similarly, and in contrast to Becker and Murphy (1992), an increase in task interdependence has an ambiguous impact on specialization. Intuitively, firms have two ways to improve coordination: by increasing task bundling or by limiting employee flexibility and adaptiveness, hence reducing the need for task bundling. As we show, the latter effect tends to dominate as tasks become very interdependent. The Tayloristic organization, for example, is characterized by both extreme specialization and extreme task interdependence. Indeed all tasks in the assembly line must be perfectly synchronized, which is why employees' actions are tightly scripted.

Finally, we show that the organizational design problem is naturally convex: the better actions are coordinated through task bundling, the more adaptive the organization chooses to be and, hence, the larger the benefits to further increasing task bundling. In the absence of any offsetting convex costs to task bundling, organizations that are "somewhat" adaptive tend to perform worse than both very rigid and very adaptive organizations. This effect is reinforced when organizations choose the quality or intensity of communication endogenously. Indeed, we show that for a wide variety of communication technologies, intensive communication, broad task assignments, and employee flexibility are complementary organizational design variables. It follows that organizations tend to be of two very distinct types: either rigid, specialized, and with limited communication or very adaptive, with broad jobs and intensive communication. These results are consistent with the fact that "new organizational practices" tend to be adopted in clusters, as has been abundantly documented in both the management and economics literature.

We have already discussed how our paper relates to the few ones that explicitly endogenize specialization and the division of labor in organizations. ${ }^{3}$ More generally, our paper contributes to the team theory literature. Starting with Marschak and Radner (1972), this literature analyzes the coordination of tasks when communication is imperfect and specialization implies that information is necessarily disperse. ${ }^{4}$ The

${ }^{3}$ Lindbeck and Snower (2000) is a more recent addition to this literature.

${ }^{4}$ A strand of this literature (Radner 1993; Bolton and Dewatripont 1994; Van Zandt 1999; Vayanos 2003) analyzes the impact of delay, rather than communication failures, on the design of organizations. 
present paper, however, is one of very few that endogenize the division of labor that causes these coordination and communication problems. Cremer (1980), building on Marschak and Radner (1972), studies the optimal grouping of technological interdependent production units but takes as given the number of units that are bundled together. ${ }^{5}$ A notable exception is the study by Geanakoplos and Milgrom (1991), who offer a partial characterization of the optimal level of task bundling in a setting à la Cremer. However, their model, in which managers have limited time to process and collect information, has implications very different from ours. More task uncertainty, for example, results in more specialization since collecting information is then more important.

Finally, our paper is consistent with a large management literature that has argued that elements of organizational design are often complementary. This notion of "strategic fit" was formalized in the economics literature by Milgrom and Roberts (1990), using the mathematics of "supermodularity." Our approach differs from theirs in that we derive this complementarity in an explicit model of production. In addition, Milgrom and Roberts focus mainly on elements of manufacturing strategy as opposed to organizational design. ${ }^{6}$ A paper that does explicitly analyze complementarities between elements of organizational design is Holmstrom and Milgrom (1994). The authors focus, however, on the complementarities between worker flexibility, high-performance incentives, and worker ownership of assets.

Outline.-The paper proceeds as follows. Section II introduces the model. Sections III and IV discuss the implications of the model for organizational design. Section V considers communication as an organizational design variable and derives the conditions for complementarity between intensive communication, broad job design, and employee flexibility. Section VI presents conclusions. Finally, in Appendix A we show that our results generalize to an extension in which each task consists of only one action, which now must be adapted to local conditions and coordinated with the actions of other tasks.

${ }^{5}$ Aoki (1986), building on Cremer (1980), separately analyzes the efficiency of vertical and horizontal coordination of interdependent tasks. While this is akin to our distinction between ex ante and ex post coordination, he does not endogenize the optimal level of task bundling.

${ }^{6}$ For example, Milgrom and Roberts (1990) focus on the complementarities between the choice of technology, capital investments, and operating systems. In an overview paper (Milgrom and Roberts 1995), they briefly discuss how some elements of human resource management policies are related to this modern manufacturing strategy, including horizontal communication and worker autonomy. They simply outline the assumptions on the cross-derivatives of the reduced-form profit function that are sufficient and necessary to guarantee the complementarity, however, without endogenizing these assumptions. 


\section{The Model}

In this section, we present a new team-theoretic model of production in which workers take actions after observing some local information and after having communicated with other workers involved in production. Organizational design determines the effectiveness of these actions through its impact on the information structure and productive efficiency of workers.

\section{A. Adaptation and Coordination}

Production, in our model, requires the combination of $n$ tasks; the profits of the organization depend on (i) how well each task is adapted to the organizational environment and (ii) how well each task is coordinated with the other tasks.

Adaptation.-Task $i$ consists of undertaking a primary action, $a^{i i}$, whose effectiveness depends on how well it is adapted to the local environment. Thus adaptation calls for the use of local information, which exclusively pertains to a particular task and can be observed only by the worker assigned to it. This local information, a random variable $\theta^{i}$ with mean $\hat{\theta}^{i}$ and a common variance $\sigma_{\theta}^{2}$, determines the optimal primary action. In particular, to achieve perfect adaptation, the primary action $a^{i i}$ should be set equal to $\theta^{i}$. The realization of the local information is independent across tasks.

Coordination.-In addition, in order to ensure that task $i$ is coordinated with all tasks $j \neq i$, the employee in charge of task $i$ must perform a string of $n-1$ actions $\left\{a^{i 1}, a^{i 2}, \ldots, a^{i n}\right\}$ that are complementary to the primary actions of tasks $j \neq i$. In particular, to achieve perfect coordination between tasks $i$ and $j$, action $a^{i j}$ of task $i$ should be set equal to the primary action $a^{i j}$.

For instance, if the organization consists of two tasks, then adaptation and coordination losses amount to

$$
\phi\left[\left(a^{11}-\theta^{1}\right)^{2}+\left(a^{22}-\theta^{2}\right)^{2}\right]+\beta\left[\left(a^{12}-a^{22}\right)^{2}+\left(a^{21}-a^{11}\right)^{2}\right],
$$

where the parameters $\phi$ and $\beta$ determine the importance of adaptation and coordination respectively.

To illustrate our modeling choices, consider the design of products and complex systems, activities in which the trade-offs between adaptability and coordination are very prevalent. For example, coordination problems are particularly daunting in the case of the development of software projects large enough to be beyond the capabilities of a single software engineer. It is for this reason that large codes are broken into several modules, each being assigned to a different team. For instance, in the development of a proprietary operating system, one module may 
be focused on the process manager, whereas another is responsible for the network access. Each module will have actions that are specific to its function, the primary actions in our framework. At the same time, though, the modules will have to be combined in one coherent whole. Software design choices geared toward the harmonious combination of these modules are the complementary actions in our setup. Uncertainty in software development springs from its nonroutine nature. Indeed, most projects are specifically tailored to the customer's needs, and each module is likely to encounter peculiarities that are unique to that project. These peculiarities have to be addressed since the code is being written by the different teams, and it is here where the trade-off between adaptability to the specific needs of the client and coordination across teams arises. ${ }^{7}$

A similar case, taken from Hammer and Champy $(2001,48)$, considers product design at Kodak:

In a parallel design process, all the parts are designed simultaneously and integrated at the end. But this method engenders its own problem: Usually, the subsystems will not fit together because, even though all the groups were working from the same basic camera design, changes-often improvements-occurred along the way but were not communicated to the other groups. Then when the camera is supposed to be ready to go to production, it's back to square one in design.

More generally, the success of organizations depends on how well they can respond to particular market conditions (demand may be higher or lower than expected) and operational conditions (a worker may be ill, an unexpected delay may occur) and how efficiently they can customize their products or services to particular consumer characteristics or changing consumer needs. Typically, the above desire for adaptation will result in coordination problems in the presence of specialization.

\section{B. Task Specialization}

An important objective of organizational design is to partition the totality of tasks into smaller jobs and assign them to specific individuals

\footnotetext{
${ }^{7}$ For a description of the coordination problems in the construction of proprietary operating systems, see Crowston (1997). The literature devoted to coordination problems in software engineering is large. See, e.g., Zmud (1980), Curtis, Krasner, and Iscoe (1988), Kraut and Streeter (1995), Faraj and Sproull (2000), and the references therein.
} 
or groups. For conciseness, we will think of the organizational problem as the assignment of jobs to workers. ${ }^{8}$

Each task is assigned to exactly one employee, but an employee may have several tasks assigned to him. We denote by $\mathcal{T}(i)$ the set of tasks bundled with task $i$. To simplify the analysis we restrict the organization of production to be symmetric; that is, all workers inside the organization have an identical number $t$ of tasks assigned to them. Thus we exclusively consider organizations in which $t \in \mathcal{J}=\{t \in \mathbb{N}$ such that $n / t \in \mathbb{N}\}$.

Task variety is costly in the usual specialization sense: as in Adam Smith's pin factory, the larger the number of tasks assigned to an employee, the lower his degree of specialization and, as a consequence, the lower his productivity. ${ }^{9}$ Concretely, the labor cost of carrying out task $i$, denoted by $h(t, \alpha)$, is increasing in the level of task bundling $t$, where the parameter $\alpha$ governs the returns to specialization. For instance, if a worker performs a broad collection of tasks, it may take him longer to complete each of them, which is costly for the organization. Alternatively, having broadly defined jobs may require more training or the hiring of more skilled agents who command higher wages.

Throughout we make use of the following notation: For any real function $f$ with support on $\mathcal{J}$, let

$$
\Delta f(\bar{t}, \underline{t})=f(\bar{t})-f(\underline{t}), \quad \Delta_{x} f(\bar{t}, \underline{t})=f_{x}(\bar{t})-f_{x}(\underline{t}),
$$

where $f_{x}$ denotes the partial derivative with respect to a parameter $x$. When we let $\bar{t}>\underline{t}$, Smithian returns to specialization imply that

$$
\Delta h(\bar{t}, \underline{t}) \geq 0, \quad \Delta_{\alpha} h(\bar{t}, \underline{t}) \geq 0,
$$

which we assume throughout.

\section{Communication}

To improve coordination between specialized tasks, workers can communicate the realization of the local information $\theta^{i}$ prior to the actual implementation of the actions. ${ }^{10}$ Such communication, however, will often be imperfect. Some reasons are the limitations on employees' ability to hold unscheduled meetings or the lack of a shared language

\footnotetext{
${ }^{8}$ The analysis of the assignment of jobs to subunits under the direction of a subunit manager is formally identical.

${ }^{9}$ Alternatively, we could assume that task variety reduces the worker's ability to observe or adapt to the local conditions, $\theta^{i}$. This case was investigated in Dessein and Santos (2003).

${ }^{10}$ Whether what is communicated is the actual realization of the local condition or the choice of the primary action itself is not key because as we show in proposition 1 , there is a one-to-one correspondence between them.
} 
that facilitates the quick transmission of information. As a result, an employee may not understand what the particular realization of the choice of a primary action by another employee implies for the corresponding complementary action under his control.

In particular, if tasks $i$ and $j$ are assigned to different employees, then with a probability $1-p$, the message concerning $\theta^{i}$ will be pure noise for the employee in charge of task $j$; thus the choice of the relevant complementary action $a^{j i}$ cannot be made contingent on it. In contrast, with a probability $p$, the agent in charge of task $j$ perfectly understands this message, and he will choose the complementary action $a^{j i}$ accordingly. We refer to $p$ as the quality of the communication channel. ${ }^{11}$

We assume that an agent never knows whether his communication with other agents was successful or not. Thus, when deciding on a primary action $a^{i i}$, he takes into account that with a probability $p$, the employee in charge of $a^{j i}$ will be influenced by his communication on $a^{i i}$. This greatly simplifies both the analysis and the exposition, and it does not affect our results qualitatively. ${ }^{12}$

\section{The Production Function}

The organization's profits are given by

$$
-\sum_{i=1}^{n} C^{i}\left(a^{1 i}, a^{2 i}, \ldots, a^{n i}, t \mid \theta^{i}\right)
$$

where

$$
C^{i}\left(a^{1 i}, \ldots, a^{n i}, t \mid \theta^{i}\right) \equiv \phi\left(a^{i i}-\theta^{i}\right)^{2}+\sum_{j \notin \mathcal{T}(i)} \beta\left(a^{j i}-a^{i i}\right)^{2}+h(t, \alpha)
$$

represents the production costs related to the primary action of task $i$.

As noted above, the effectiveness of the primary action $a^{i i}$ depends on how close it is set to the local circumstances $\theta^{i}$, capturing the need for adaptation of task $i$ to the organizational environment. The effectiveness of the complementary actions $a^{j i}$ depends on how close they are set to the choice of $a^{i i}$, capturing the need for coordination between the primary action of task $i$ and the other tasks. The parameters $\phi$ and $\beta$ determine the importance of adaptation and coordination respectively.

It is useful to return here to our modeling choices concerning adaptation and coordination. Workers can communicate only imperfectly.

\footnotetext{
${ }^{11}$ Throughout, and given that we restrict the analysis to symmetric organizations, we assume that $p$ is common across channels.

${ }^{12}$ It further implies that it does not matter for our results whether the probability of a successful communication between tasks $i$ and $j \notin \mathcal{T}(i)$ is correlated with that between tasks $i$ and $k \notin \mathcal{T}(i)$
} 
Hence, in the presence of task specialization, adaptation to local circumstances puts at risk the harmonious coordination of specialized tasks. This trade-off between coordination and adaptation, however, completely disappears if all tasks are assigned to one worker. Indeed notice in expression (4) that in what concerns the costs associated with task $i$, the organization suffers coordination costs only for those tasks not bundled with tasks $i, j \notin \mathcal{T}(i)$. Perfect adaptation and coordination can then be simultaneously achieved.

Another modeling approach, studied in Appendix A, is to have only primary actions that must be adapted both to local circumstances and to the primary actions of other tasks. This approach, however, introduces a technological trade-off between adaptation and coordination, which is present even in the absence of any division of labor. In our model, this trade-off is purely organizational, arising exclusively out of design choices involving specialization and communication. As we show in Appendix A, our main insights are robust to this alternative approach.

\section{E. Timing}

The timing of our model goes as follows:

i. Organizational design stage: The organization determines the number of tasks $t$ per agent.

ii. The local circumstances $\theta^{i}, i=1,2, \ldots, n$, are realized and observed by the employee in charge of task $i$.

iii. Communication stage: Workers communicate the realizations of local information, and with an independent probability $p$, these communications are successful.

iv. Action stage: For all $i=1,2, \ldots, n$, the employee in charge of task $i$ chooses actions $a^{i j}, j=1,2, \ldots, n$, in such a way as to maximize the objective function (3), subject to his information constraints.

\section{Organizational Actions and Performance}

Production requires the combination of $n$ tasks; each of them involves the choice of a primary action that needs to be adapted to the local circumstances and $n-1$ complementary actions that must be coordinated with the $n-1$ primary actions of other tasks. We start by characterizing the choice of these actions as a function of a particular organizational design $t$ in proposition 1 and then evaluate the costs associated with that particular organizational design in proposition 2.

Proposition 1. Given a level of task bundling $t$ and communication quality $p$ and faced with a vector of local circumstances $\left(\theta^{1}, \theta^{2}, \ldots\right.$, 
$\theta^{n}$ ), employees optimally choose the following primary and complementary actions:

$$
a^{i i}(t)=\hat{\theta}^{i}+\left[\frac{\phi}{\phi+\beta(n-t)(1-p)}\right]\left(\theta^{i}-\hat{\theta}^{i}\right)
$$

and

$$
a^{j i}(t)= \begin{cases}a^{i i}(t) & \text { when task } j \text { learns } \theta^{i} \\ \hat{\theta}^{i} & \text { when task } j \text { does not learn } \theta^{i} .\end{cases}
$$

Expression (5), which gives the choice of the primary action, has two terms. The first one is the expected value of the local circumstances, which, trivially, is also equal to the expected value of the primary action. The second term reflects the adaptation to the local information, $\theta^{i}$, which is limited by the term $\phi /[\phi+\beta(n-t)(1-p)] .{ }^{13}$

To capture how closely agents tailor the primary actions to local circumstances, we introduce the concept of employee flexibility, which is formally defined as the covariance between primary actions and the corresponding local information:

$$
\sigma_{a \theta}(t)=\operatorname{Cov}\left[a^{i i}(t), \theta^{i}\right] \equiv\left[\frac{\phi}{\phi+\beta(n-t)(1-p)}\right] \sigma_{\theta}^{2} .
$$

Thus $\sigma_{a \theta}(t)$ characterizes how strictly employees adhere to an ex ante plan of action or, in contrast, tailor their actions to local circumstances.

Intuitively, the level of employee flexibility $\sigma_{a \theta}(t)$ is increasing in the importance of local adaptation, $\phi$, decreasing in the importance of maintaining coordination in the organization, $\beta$, and increasing in the variability of the local circumstances, $\sigma_{\theta}^{2}$. It is decreasing in the term $\beta(n-t)(1-p)$, which measures the limits to adaptation that result from the need to maintain some coordination with other tasks. As one might expect, an increase in task bundling $t$ results in primary actions that are more adaptive to the local information since now the worker can rely on a perfect coordination with the additional tasks under his control; that is,

$$
\Delta \sigma_{a \theta}(\bar{t}, \underline{t}) \geq 0
$$

where the notation $\Delta \sigma_{a \theta}(\bar{t}, \underline{t})$ was introduced in (1). Similarly, increasing the quality of the communication channels $p$ naturally improves coordination since now complementary actions are based on better information. This results in more adaptive primary actions because the em-

\footnotetext{
${ }^{13}$ Notice that, given (5), there is a one-to-one correspondence between $a^{i i}$ and $\theta^{i}$, so the successful communication of one is equivalent to the successful communication of the other.
} 
ployees can feel confident that these actions are more likely to be understood by other agents in the organization. Clearly $\sigma_{a \theta}(t)=\sigma_{\theta}^{2}$ whenever $t=n$ and all tasks are bundled in a single job or $p=1$ and communication is perfect. The reason is that in either case the tradeoff between adaptation and coordination disappears and agents can fully adapt to the local information. Given proposition 1, the next proposition provides a tractable expression for this expected profit function, which is key in the characterization of organizational design.

Proposition 2. The expected profits for a given level of task bundling $t$ are given by

$$
\Pi(t) \equiv-E\left[\sum_{i} \min _{\bar{a}^{i}} C^{i}\left(\bar{a}^{i}, t \mid \theta_{i}\right)\right]=n \phi\left[\sigma_{a \theta}(t)-\sigma_{\theta}^{2}\right]-n h(t, \alpha),
$$

where $\bar{a}^{i}=\left(a^{1 i}, a^{2 i}, \ldots, a^{n i}\right)$.

The first term in (9) captures how well the organization is adapted and coordinated and is increasing in $t$. The second term, reflecting the returns to specialization in labor costs, is decreasing in $t$. Our purpose next is to characterize

$$
t^{*}=\arg \max _{t \in \mathcal{J}} \Pi(t)
$$

as a function of the parameters of the model, $\phi, \alpha, \sigma_{\theta}^{2}, \beta$, and $p$.

\section{Organizational Design}

\section{A. The Trade-off between Specialization and Adaptation}

As an example of the type of comparative statics that are of interest in what follows, consider the case of environmental uncertainty, $\sigma_{\theta}^{2}$. The management literature often attributes the trend toward new workplace practices, including broader job descriptions, to the more volatile business environment that organizations face. Consistent with this observation, our model predicts that an increase in the environmental uncertainty produces a decrease in the extent of specialization. Indeed, given proposition 2, it follows trivially from (9) that

$$
\Delta_{\tau} \Pi(\bar{t}, \underline{t}) \geq 0 \quad \text { for } \tau \in\left\{\phi, \sigma_{\theta}^{2},-\alpha\right\}
$$

that is, the returns to bundling tasks into broader job descriptions increase with the variance of local conditions, $\sigma_{\theta}^{2}$, and the importance of 
adaptation, as measured by $\phi$, and decrease in the returns to specialization, $\alpha .{ }^{14}$ Proposition 3 states this formally.

Proposition 3. Task specialization is decreasing in the importance of adaptation, $\phi$, and the variance in local circumstances, $\sigma_{\theta}^{2}$, but increasing in the returns to specialization, $\alpha$.

Corollary 1. The level of employee flexibility $\sigma_{a \theta}\left(t^{*}\right)$ is increasing in the parameters $\sigma_{\theta}^{2}$ and $\phi$ and decreasing in the parameter $\alpha$.

Corollary 1 follows trivially from proposition 3 , and it is given without proof. Intuitively, organizations can coordinate their activities ex ante by letting employees tightly stick to some preagreed course of action. This type of coordination can be done even in the presence of extensive division of labor, since no communication between workers is required. Alternatively, employees may be given substantial flexibility to adapt their tasks to local circumstances. This requires ex post coordination, using communication and broad job definitions. Since communication is imperfect, however, this may result in substantial coordination problems when individuals are very specialized. A direct implication is that specialization is constrained by the organization's desire to be responsive and adaptive to its environment. An increase in the importance of adaptation, or the variance in local circumstances, then results in broader job definitions and a less extensive division of labor. Conversely, an increase in the returns to specialization reduces the flexibility employees receive to carry out their job.

\section{B. The Tenuous Trade-off between Coordination and Specialization}

We now consider the impact of two parameters that directly affect the trade-off between coordination and specialization: $\beta$, which characterizes the task interdependence or the importance of coordination, and $p$, which characterizes the quality and effectiveness of communication.

Task interdependence.-Adam Smith's famous observation that "the division of labor is limited by the extent of the market" has been challenged by both the management literature and economists such as Becker and Murphy (1992). These two strands of the literature have emphasized that, perhaps more important, specialization is mainly constrained by the need to coordinate activities. In particular, a straightforward comparative static prediction in Becker and Murphy's study is

\footnotetext{
${ }^{14}$ With some abuse of terminology we refer to $\Delta \Pi(\bar{t}, \underline{t})$ as the returns to broadening job descriptions from $\underline{t}$ tasks per job to $\bar{t}$ tasks per job, though, more formally, it should be defined as $\Delta \Pi(\bar{t}, \underline{t}) /(\bar{t}-\underline{t})$.
} 
that as coordination costs increase, one should see less specialization. ${ }^{15}$ Taking into account the three-way trade-off between coordination, specialization, and adaptation yields a much more subtle picture.

Indeed, it can be shown that the returns to increasing task bundling from $\underline{t}$ to $\bar{t}$ are nonmonotonic in $\beta$ :

$$
\Delta_{\beta} \Pi(\bar{t}, \underline{t}) \leq 0 \quad \text { if and only if } \phi \leq \beta(1-p)(n-\underline{t})^{1 / 2}(n-\bar{t})^{1 / 2} .
$$

Thus a higher task interdependence parameter, $\beta$, decreases the returns to specialization when $\phi$ is large and adaptation is important. In this case an increase in $\beta$ is likely to be accompanied by broader job descriptions, which is the result in Becker and Murphy (1992). Instead, when $\phi$ is small, an increase in task interdependence boosts the returns to specialization and may result in narrower jobs.

Intuitively, an increase in task interdependence affects two margins. First, for a given level of employee flexibility, it becomes more important to improve coordination by increasing task bundling. This is the effect emphasized in the literature. Second, it pushes the organization to constrain employee flexibility and become less adaptive. This reduces the need for task bundling. The latter effect often dominates when tasks become very interdependent. Indeed, the organization may then choose to eliminate any employee flexibility and fully specialize its employees $\left(t^{*}=1\right)$ since task bundling has no benefit then. Similarly, if $\beta$ is very small and tasks are virtually independent, task coordination can be neglected and it is optimal to have full task specialization $\left(t^{*}=1\right)$. The next proposition characterizes the optimal level of task bundling as a function of the task interdependence parameter $\beta .{ }^{16}$

Proposition 4. Assume that $h(n, \alpha)<\infty$. (a) Then given $\alpha$, there exists a unique $\hat{\phi}$ such that

$$
\lim _{\beta \rightarrow \infty} t^{*}= \begin{cases}n & \text { if } \phi>\hat{\phi} \\ 1 & \text { if } \phi<\hat{\phi},\end{cases}
$$

and $\hat{\phi}$ is increasing in $\alpha$. (b) Let $t^{*}(\beta)$ be the optimal level of task bundling given $\beta$, and $\hat{\beta}=\min \left\{\arg \max _{\beta} t^{*}(\beta)\right\}$. Then if $\phi<\hat{\phi}, t^{*}$ is decreasing in $\beta$ for $\beta \geq \hat{\beta}$.

\footnotetext{
${ }^{15}$ Becker and Murphy do not model the sources of the coordination costs that specialization would bring, however, nor any form of communication between the parties, so their model has limited organizational design implications. They emphasize instead the impact of growth in human capital on the extent of specialization.

${ }^{16}$ There is some supportive empirical evidence that more task interdependence leads to more specialization, not less. In a study of Japanese vs. U.S. organizational practices, Lincoln, Hanada, and McBride (1986) show that workers in Japanese firms are less specialized than their U.S. counterparts. In order to find the determinants of specialization, they regress task specialization on several variables, of which task interdependence is one (see their table 3.) The coefficient on task interdependence is positive and significant at the 10 percent level. The table is partly reproduced in Dessein and Santos (2003).
} 


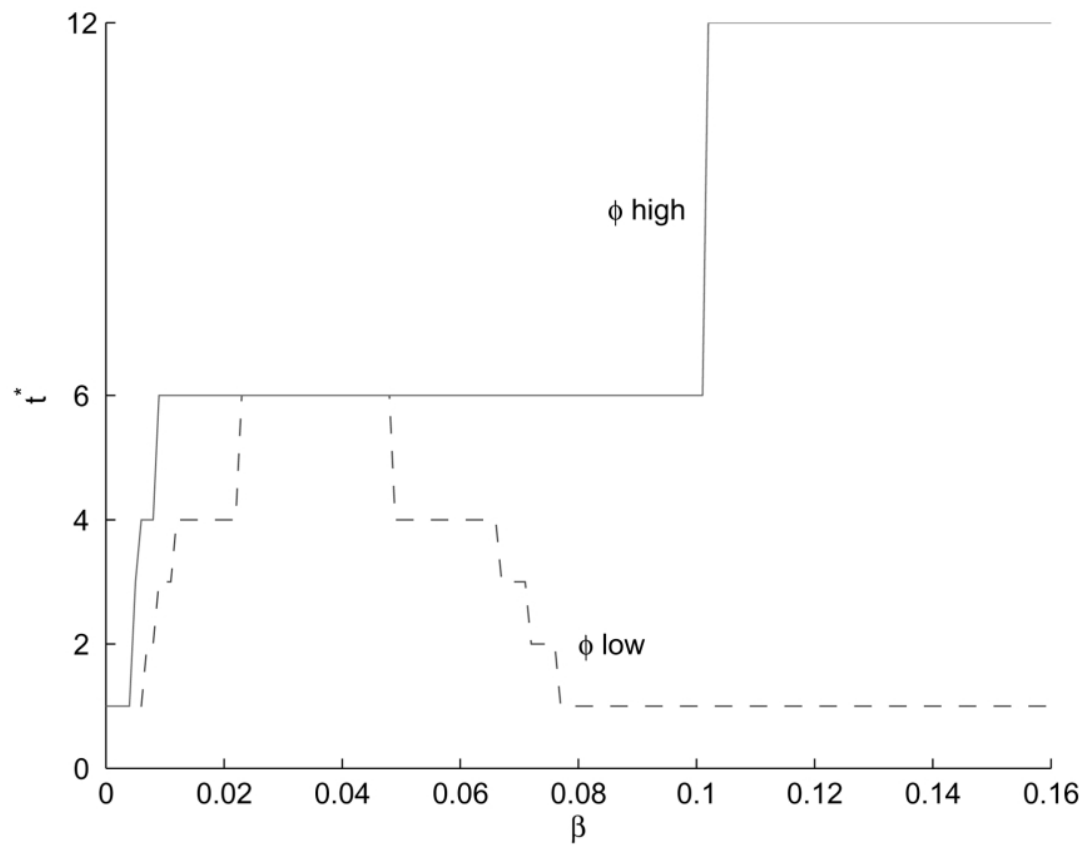

Fig. 1.-Optimal task bundling $t^{*}$ as a function of the importance of coordination, $\beta$, for the case considered in example 1. The continuous line, denoted $\phi$ high, shows $t^{*}$ when the importance of adaptation is high, $\phi>\hat{\phi}$, where $\hat{\phi}$ is given in proposition 4 ; the dashed line, denoted $\phi$ low, shows $t^{*}$ as a function of $\beta$ when the importance of adaptation is low, $\phi<\hat{\phi}$.

Proposition 4 formalizes the intuition described above: As task interdependence increases without bound, the organization becomes fully specialized when the costs of not adapting to the local circumstances, as captured by $\phi$, are below a certain cutoff value $\hat{\phi}$. In this case, $t^{*}$ is increasing in task interdependence whenever $\beta$ is below a certain cutoff value, $\hat{\beta}$, as Becker and Murphy (1992) would have it, whereas it is decreasing afterward. Thus the organization goes from an ex post coordination mode to an ex ante coordination one, which is rigid and nonadaptive. Example 1 next illustrates these findings.

EXAMPLE 1. Assume that the number of tasks is $n=12$, the quality of communication is $p=0$, and the variance of local conditions is $\sigma_{\theta}^{2}=2$. The specialization costs are

$$
h(t, \alpha)=\frac{\alpha}{1+(n-t)} \quad \text { with } \alpha=1 .
$$

It can be easily calculated that in this case $\hat{\phi}=.45$. Figure 1 plots the level of task bundling, $t^{*}$, as a function of $\beta$ for an organization for 
which $\hat{\phi}<\phi=1.45$ (the thick line) and a second one for which $\hat{\phi}>$ $\phi=.23$ (the dashed line). For the organization for which $\phi>\hat{\phi}$, adaptation is sufficiently important that $t^{*}$ is always increasing in $\beta$. Instead for the second organization, where $\phi<\hat{\phi}$, the extent of specialization is nonmonotone in the importance of coordination.

Communication quality.-Perhaps it is most natural to coordinate specialized activities through communication. Employees spend a substantial amount of their time communicating with each other in the workplace: Meetings, conference calls, voice mails, and writing and reading electronic mail dominate the modern professional environment. An important question, therefore, is how improvements in communication technology or differences in the ease of communication affect specialization. For instance, if, for logistical or epistemological reasons, the transmission of information is easier in certain economic processes or activities, do we expect to see more or less specialization?

Bolton and Dewatripont (1994) and Garicano (2000), among others, have addressed this issue in different settings. ${ }^{17}$ These authors identify an important trade-off between specialization and communication costs, namely, that one should expect to see more specialization as communication costs decrease and coordination becomes easier. This logic, however, ignores that organizations take advantage of lower communication costs or improved communication to become more adaptive, requiring more ex post coordination. Just as a price decrease may increase overall expenditures because demand is elastic, improvements in communication quality may therefore increase the need for task bundling.

In very much the same way as a change in $\beta$, an improvement in the quality of communication affects two margins. First, for a given level of employee flexibility, it will be easier to coordinate specialized activities, reducing the need for task bundling. This is the effect emphasized by Bolton and Dewatripont (1994) and Garicano (2000). Second, as coordination through communication improves, the organization will find it optimal to increase employee flexibility and become more adaptive. This favors more task bundling in order to reduce coordination failures.

As we show next, when communication channels are very poor, task bundling then often increases as communication technology improves. Intuitively, when communication is very poor, organizations often choose to limit the need for ex post coordination by limiting employee flexibility. In the latter case, improvements in communication will typically be complementary to broader task assignments. In particular, they

\footnotetext{
${ }^{17}$ Bolton and Dewatripont (1994) consider the returns to division of labor in information processing. Garicano (2000) considers vertical specialization in knowledge acquisition, where some agents may specialize in solving more difficult problems.
} 


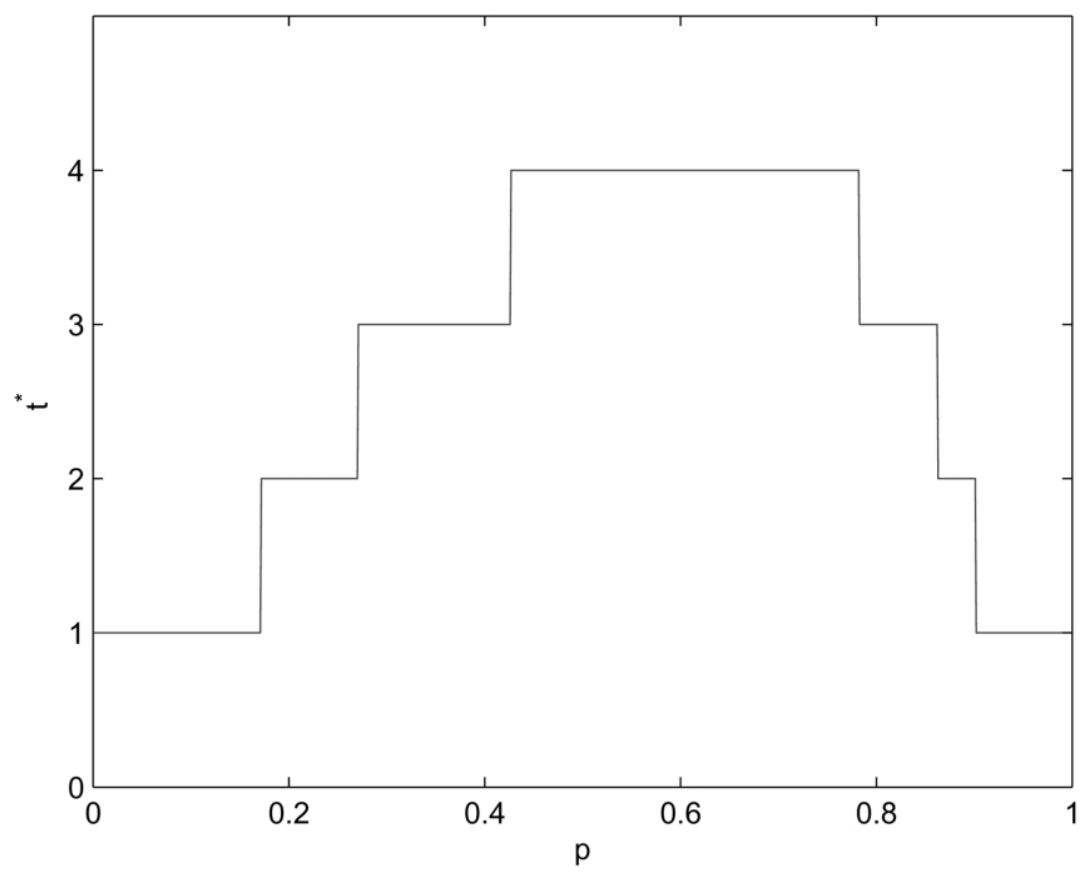

FIG. 2.-Optimal task bundling, $t^{*}$, as a function of the quality of communication, $p$, for the case considered in example 2.

may transform this very rigid and specialized organization into a flexible organization characterized by substantial employee flexibility and broad task assignments. As communication technology further improves, however, the benefits of task bundling in terms of reduced coordination failures become smaller and smaller. Task bundling and improvements in communication technology then become substitutes, and task specialization increases again. The next example illustrates the nonmonotonicity of $t^{*}$ with respect to $p$.

EXAMPLE 2. As before we consider the case of a production process in which $n=12$ and $\sigma_{\theta}^{2}=2$. Also, $\beta=1$ and $\phi=3$. Finally, the specialization costs are given by

$$
h(t, \alpha)=\alpha \frac{t}{n-t},
$$

where $\alpha=1$. Figure 2 shows the level of task bundling, $t^{*}$, as a function of $p$ for this economy. As can be seen in the figure, for sufficiently low values of $p$, improvements in communication quality, as measured by $p$, lead to more task bundling, not less. Only when $p$ is close to one, further 
improvements in the quality of communication result in the standard increase in specialization.

Formally, and from the same steps to derive (10) above,

$$
\Delta_{p} \Pi(\bar{t}, \underline{t}) \geq 0 \quad \text { if and only if } \phi \leq \beta(1-p)(n-\underline{t})^{1 / 2}(n-\bar{t})^{1 / 2} .
$$

Hence improvements in the communication quality boost the returns to increasing task bundling from $\underline{t}$ to $\bar{t}$ when the coordination costs, $\beta$, are high, the quality of the communication, $p$, remains low, or when, even after the additional task bundling, $\bar{t}$ stays low as well. The following proposition characterizes the optimal degree of task bundling as a function of $p$.

Proposition 5. (a) Given $\alpha$, there exists a unique $\hat{\phi}$ such that

$$
\lim _{p \rightarrow 0} t^{*}=1 \quad \text { if and only if } \phi<\hat{\phi},
$$

where $\hat{\phi}$ is increasing in $\alpha$. (b) Let $t^{*}(p)$ be the optimal level of task bundling given $p$ and $\hat{p}=\min \left\{\arg \max _{p} t^{*}(p)\right\}$. Then if $\phi<\hat{\phi}, t^{*}$ is increasing in $p$ for $p \leq \hat{p}$.

\section{Convexities in Organizational Design}

An important feature of the organizational problem under study is that it is a highly convex one. Indeed, let $\underline{t}<\bar{t}<\overline{\bar{t}} \in \mathcal{J}$. Then from (8),

$$
(\overline{\bar{t}}-\bar{t})^{-1} \Delta \sigma_{a \theta}(\overline{\bar{t}}, \bar{t})>(\bar{t}-\underline{t})^{-1} \Delta \sigma_{a \theta}(\bar{t}, \underline{t}) .
$$

Since, from (9), profits are linear in the level of employee flexibility $\sigma_{a \theta}(t)$, it follows that as long as $h(t, \alpha)$ is not "too convex," organizations exhibit increasing returns to task bundling. Therefore, even when a change from $\underline{t}$ to $\bar{t}$ reduces profits, a change from $\underline{t}$ to $\overline{\bar{t}}$ may actually increase profits. Intuitively, when jobs are very specialized, employees receive little flexibility, and hence there is not much value to increasing task bundling. The broader the job description, however, the more flexibility employees receive, which in turn increases the returns to further increasing task bundling.

A direct consequence is that, in the latter case, organizations tend to be of two distinct types. Either they are very rigid and specialized and rely on ex ante coordination or they will exhibit substantial employee flexibility and rely on extensive ex post coordination through task bundling. It follows that organizations may be very sensitive to small changes in the importance of adaptation, $\phi$, or task uncertainty, $\sigma_{\theta}^{2}$, which can result in a dramatic and discrete organizational change. Similarly, from (10) and (11), starting with an organization with $\underline{t}$ tasks per job, a higher $\beta$ or smaller $p$ may reduce the returns to increasing the tasks per job 
to $\bar{t}$ but may actually increase the returns to going to an organization with even broader job descriptions $\overline{\bar{t}}>\bar{t}$. Hence, small changes in task interdependence or small improvements in communication technology can result in big changes in organizational design.

\section{Communication as an Organizational Choice}

In the previous section we showed how an exogenous improvement in communication affects specialization in a nontrivial way. This section shows that when organizations choose the quality or intensity of communication endogenously, broad task assignments, employee flexibility, and intensive communication are complementary organizational choices. We start by endogenizing communication quality in subsection $A$. Subsection $B$ then discusses the complementarity between task bundling and investments in communication quality and generalizes the unambiguous comparative static predictions obtained in the previous section. Finally, subsection $C$ illustrates the robustness of the tenuous trade-offs between specialization and coordination, and specialization and communication technology.

\section{A. Endogenizing Communication Quality}

We assume that the organization can achieve the level of communication quality $p$ at the cost $g(t, p, \delta)$ with

$$
g_{p} \geq 0, \quad g(t, 0, \delta)=g_{p}(t, 0, \delta)=0, \quad \lim _{p \rightarrow 1} g_{p}=\infty .
$$

We interpret $g(p, t, \delta)$ as the opportunity cost to the organization of having workers engaged in regular meetings, conference calls, and electronic mail conversations rather than in production itself. Similarly, the organization can improve communication channels by job rotation or team events or by hiring employees with knowledge or skills that span across job boundaries. In $g(p, t, \delta), \delta$ parameterizes communication technologies, and we assume that

$$
g_{\delta}>0, \quad g_{p \delta}>0 .
$$

The functional form of organizational profits can be trivially updated to account for the communication cost as

$$
\Pi(t, p)=n \phi\left[\sigma_{a \theta}(t, p)-\sigma_{\theta}^{2}\right]-n h(t, \alpha)-g(t, p, \delta),
$$

where $\sigma_{a \theta}(t, p)$, the level of employee flexibility, is given by (7) and is now a function of $p$ as well.

Modeling the communication costs can be done in various ways, but in order to establish our results, it is enough to impose one additional 
assumption on these costs. Let

$$
\tilde{g}(t, p, \delta) \equiv\left[\frac{2}{n(n-t)}\right] g(t, p, \delta),
$$

which is the communication cost per unbundled task pair $(i, j)$ with $j \notin \mathcal{T}(i)$. Then we make the following assumption.

Assumption $1 . \quad \Delta_{p} \tilde{g}(\bar{t}, \underline{t})=\tilde{g}_{p}(\bar{t}, p, \delta)-\tilde{g}_{p}(t, p, \delta) \leq 0$.

Assumption 1 says that the marginal cost (per unbundled task pair) of increasing the quality of the communication is weakly lower when agents are less specialized, and it is verified for at least three reasonable communication technologies: task-to-person, task-to-task, and person-toperson. We elaborate on these different forms of communication next.

Perhaps the most natural way to introduce communication costs in our model is to assume that agents incur a cost $m(p, \delta)$ each time they communicate the choice of a primary action to another agent. Since each agent must communicate the primary action of each assigned task to $(n / t)-1$ other agents, this yields a total communication cost $g(t, p$, $\delta)=n[(n / t)-1] m(p, \delta)$. Assuming $m_{p}>0$, we can immediately verify that $\Delta_{p} \tilde{g}(\bar{t}, \underline{t})<0$. We will refer to this technology as task-to-person communication.

Alternatively, the choice of a primary action $a^{i i}$ may need to be communicated $n-t$ times. This assumption is realistic if the way a particular task is carried out cannot be easily summarized and affects different tasks differently. For each task pair, the agents in charge must then discuss how to coordinate these two tasks. ${ }^{18}$ Thus a worker in charge of tasks $j$ and $k$ may understand what the choice of $a^{i i}$ means for task $j$ and set $a^{j i}=a^{i i}$. But because communication is task-to-task, he may not understand what it means for task $k$, so he sets $a^{k i}=\hat{\theta}^{i}$. Since there are now $n(n-t)$ as opposed to $n[(n / t)-1]$ communication channels, this yields $g(t, p, \delta)=n(n-t) m(p, \delta)$ and $\Delta_{p} \tilde{g}(\bar{t}, \underline{t})=0$.

Finally, communication can be person-to-person. This is the relevant communication technology when employees may need to get to know each other better, socialize, establish trust, or simply find a time to meet in order to discuss all their coordination needs. In this case, the number of channels is the number of employee pairs, which is $.5(n / t)[(n / t)-$ $1]$. Thus now $g(t, p, \delta)=.5(n / t)[(n / t)-1] m(p, \delta)$. Again, one can immediately verify that $\Delta_{p} \tilde{g}(\bar{t}, \underline{t})<0$.

Notice that under task-to-person and person-to-person communication, the marginal cost to improving the coordination between two unbundled tasks, $\tilde{g}_{p}(t, p, \delta)$, is decreasing in the level of task bundling.

\footnotetext{
${ }^{18}$ For example, in the case of product development at Kodak, improvements implemented by the shutter designers are likely to have different implications for the camera body designers and the film mechanism designers.
} 
These technologies thus introduce a mechanical complementarity between $p$ and $t$. In contrast, under task-to-task communication, any observed complementarity between $p$ and $t$ is purely organizational since $\Delta_{p} \tilde{g}(\bar{t}, \underline{t})=0$. To emphasize such organizational complementarities, our examples below will focus on task-to-task communication.

We next characterize

$$
\left(t^{*}, p^{*}\right)=\arg \max _{t \in \mathcal{J}, p \in[0,1]} \Pi(t, p)
$$

as a function of the parameters of the model, $\phi, \alpha, \sigma_{\theta}^{2}, \beta$, and $\delta$.

\section{B. Complementarities in Organizational Design}

We now show how for any given communication technology $g(t, p, \delta)$ that satisfies assumption 1 , broad job assignments, $t$, and investments in communication quality, $p$, are complementary instruments of organizational design at the optimum. Indeed, since task bundling improves coordination, employees with broader jobs have more flexibility in carrying out their tasks. This in turn increases the need to improve communication between those tasks that are not yet bundled. Similarly, when two tasks become better coordinated because of increased time spent on communication, employees are optimally given more flexibility, increasing the benefits of bundling each of these tasks with other unbundled tasks.

But, how do investments in improved communication between two unbundled tasks affect the incentives to bundle these two tasks into one job? On the one hand, the higher these investments in communication, the more the organization saves on communication costs by bundling tasks $i$ and $j$. For example, agents may be forced to spend half of their time in meetings in order to achieve a smooth coordination. This can be avoided if one agent carries out both tasks $i$ and $j$. On the other hand, higher investments in communication quality also reduce the benefits of making coordination "perfect" through bundling. As shown in Appendix B, when $p$ is optimally chosen, these two effects cancel each other; thus the only remaining effect of more intensive communication between tasks $i$ and $j$ is that of increased incentives to bundle $i$ or $j$ with a third task $k .{ }^{19}$ Below we return to the complementarity between communication quality and task bundling more formally using the language of supermodularity.

A direct consequence of this complementarity is that we will see more task bundling if the organization can simultaneously improve commu-

\footnotetext{
${ }^{19}$ It is in this sense that the complementarity between task bundling and the quality of the communication channels holds at the optimum, but not necessarily for values of $p$ that are below the optimal one.
} 
nication between unbundled tasks. Similarly, the choice variables $p$ and $t$ will move up or down together in response to changes in exogenous parameters, yielding a number of robust comparative static predictions, which we formalize in proposition 6 below. To focus on interior solutions, we make the following assumption, which guarantees that there is always a unique (interior) solution for the quality of communication.

Assumption 2. The expected profit function $\Pi(t, p)$ is strictly quasiconcave in $p .^{20}$

Proposition 6. Given assumptions 1 and 2, task bundling, $t^{*}$, and the quality of communication channels, $p^{*}$, are increasing in the parameters $\sigma_{\theta}^{2}$ and $\phi$ and decreasing in the parameter $\alpha$.

Corollary 2. The level of employee flexibility $\sigma_{a \theta}\left(t^{*}, p^{*}\right)$ is increasing in the parameters $\sigma_{\theta}^{2}$ and $\phi$ and decreasing in the parameter $\alpha$.

Proposition 6 generalizes the comparative static results of proposition 3 for the case in which communication is endogenous. Thus our model predicts that if adaptation to local circumstances becomes more important or the variability of this local environment increases, this will result in more employee flexibility, broader job definitions, and better horizontal communication. ${ }^{21}$ Similarly, because of this complementarity, a decrease in the returns to specialization results not only in broader job descriptions but also in more intensive horizontal communication.

Taken together, the complementarity between $p$ and $t$ and the convexity of the profit function in $t$ for those values of $t$ for which $h(t, \alpha)$ is not too convex imply that organizations tend to be of two distinct types: either very rigid, with extensive specialization and limited communication, or very adaptive, with broad tasks and intensive communication between unbundled tasks. The next example illustrates the impact of the complementarity between task bundling and the optimized level of communication quality on organizational design.

EXAMPLE 3. Assume that communication is task-to-task so that

${ }^{20}$ Obviously, this condition will always be verified whenever the expected profit function is strictly concave. A sufficient condition for strict concavity is that $2 \beta^{2} \sigma_{\theta}^{2}<\phi g_{p p}(t, p, \delta)$, which will always be verified whenever $\phi$ is large enough or $\beta$ is small enough.

${ }^{21}$ There is empirical support for the proposition that an increase in the variability of the local environment leads to the adoption of new workplace practices. For example, Van de Ven, Delbecq, and Koenig (1976) study the organizational modes of 197 formal work units of an employment security agency depending on whether these units face a high or low degree of task uncertainty. They find that those work units facing high task uncertainty adopt group coordination modes that consist of extensive horizontal communication in the form of scheduled and unscheduled meetings and make little use of what they refer to as impersonal coordination modes that consist of the use of rules and procedures; the opposite is true for those units facing low task uncertainty (these results are reported as well in tables 4 and 5 in Dessein and Santos [2003]). For additional evidence, see Argote (1982) and Osterman (1994). 


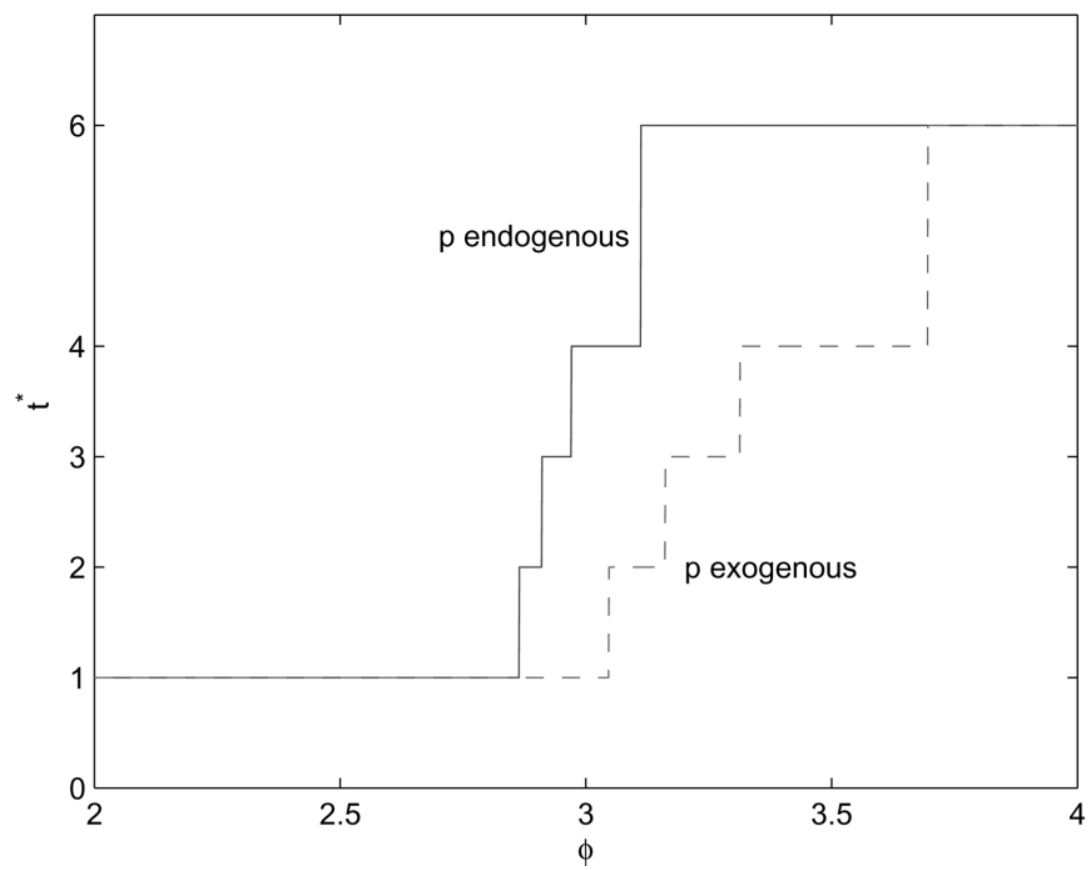

Fig. 3.-Optimal task bundling, $t^{*}$, as a function of the importance of adaptation, $\phi$, for the case considered in example 3 . The thick line, denoted $p$ endogenous, is the level of task bundling when the quality of communication, $p^{*}$, is endogenous. The dashed line, denoted as $p$ exogenous, is the level of task bundling when the quality of communication is kept fixed at its optimal level for $\phi=2$.

$g(t, p)=n(n-t) m(p, \delta)$ and

$$
m(p, \delta)=\delta\left(\frac{p^{1+\eta}}{1+\eta}\right),
$$

with $\delta=1.85$ and $\eta=1.5$. Also, the coordination parameter is $\beta=1$. The specialization costs $h(t, \alpha)$ are as in example 2. Finally, as before, $\sigma_{\theta}^{2}=2$ and $n=12$. The thick line in figure 3 , labeled " $p$ endogenous," shows $t^{*}$ as a function of $\phi \in(2,4)$ when the quality of communication, $p$, is optimally chosen. The line labeled " $p$ exogenous," the dashed line, denotes $t^{*}$ as a function of $\phi \in(2,4)$ when instead the quality of horizontal communication is kept fixed at its optimal level for $\phi=2$. As illustrated in the figure, because of the complementarity between $t$ and $p^{*}$, the returns to increasing task bundling are much larger if the organization can simultaneously improve communication between unbundled tasks. Similarly, we see intermediate values of specialization for a much smaller range of $\phi$ 's if $p$ is endogenous. 
An important empirical implication is thus that there should be a positive cross-sectional correlation between task bundling and the use of communication (and, in general, investments in information technology). Brynjolfsson and Hitt (1998, table 2a) provide substantial evidence that this is indeed the case. ${ }^{22}$ In particular, they find that the use of self-managed teams and broad job descriptions correlates positively with several measures of information technology, such as the fraction of employees who use e-mail and have access to general-purpose computers and the total purchase value of computer equipment. ${ }^{23}$

Complementarity and supermodularity.-To conclude this subsection, we discuss how the complementarity between $p$ and $t$ at the optimum can be expressed in terms of supermodularity of the profit function $\Pi(t$, $p$ ). Roughly speaking, a function is supermodular if the returns to increasing one of the arguments are higher the higher the other arguments. Supermodularity then formalizes the idea of "complementarity" or "fit" among choice variables and allows for unambiguous comparative statics. ${ }^{24}$ However, $\Pi(t, p)$ is not supermodular on the full support of its variables. In particular, if the quality of communication is (inefficiently) low, broader task assignments often become more attractive as communication deteriorates: $p$ and $t$ are typically substitutes for $p \ll p^{*}$. The complementarity between $p$ and $t$ holds only for optimized levels of communication. Therefore, in order to prove proposition 6, Appendix B considers the optimized value of profits with respect to $p$, where $p$ is restricted by a lower bound $\hat{p}$,

$$
\pi(t, \hat{p})=\max _{p \geq \hat{p}} \Pi(t, p)
$$

and shows that $\pi(t, \hat{p})$ is supermodular in $t, \hat{p}$, and any parameter $\tau \in$ $\left\{\sigma_{\theta}^{2}, \phi,-\alpha\right\}$. Obviously, this change of variables leaves the optimal value of $t$ unchanged. Moreover, if for any fixed values of the other variables $p^{*}$ is unique, the highest optimal value of $\hat{p}$ equals $p^{*}$.

\footnotetext{
${ }^{22}$ Similarly, in a well-known study of specialty steel finishing lines, Ichniowski, Shaw, and Prennushi (1995, table 2; 1997) show how the use of formal and informal teams (a proxy for intensive communication) is strongly correlated with broad job assignments. Consistent with our assumption that task specialization reduces labor costs, they also find that narrow job assignments are correlated with less off-the-job training of workers and a less selective recruiting process. They interpret their findings as showing a strong complementarity between new workplace practices.

${ }^{23}$ Bresnahan et al. (2002) provide additional evidence for this complementarity, as well as the link with the demand for high-skilled labor. Similar results are obtained by Coutrot (1996) on French data.

${ }^{24}$ In particular, if a function of a vector of choice variables $y=\left(y^{1}, \ldots, y^{k}\right)$ and an exogenous parameter $\tau$ is supermodular, then the maximizers $y^{*}(\tau)$ will be monotone nondecreasing in the parameter $\tau$. For excellent discussions on supermodularity, see Milgrom and Roberts $(1990,1995)$.
} 

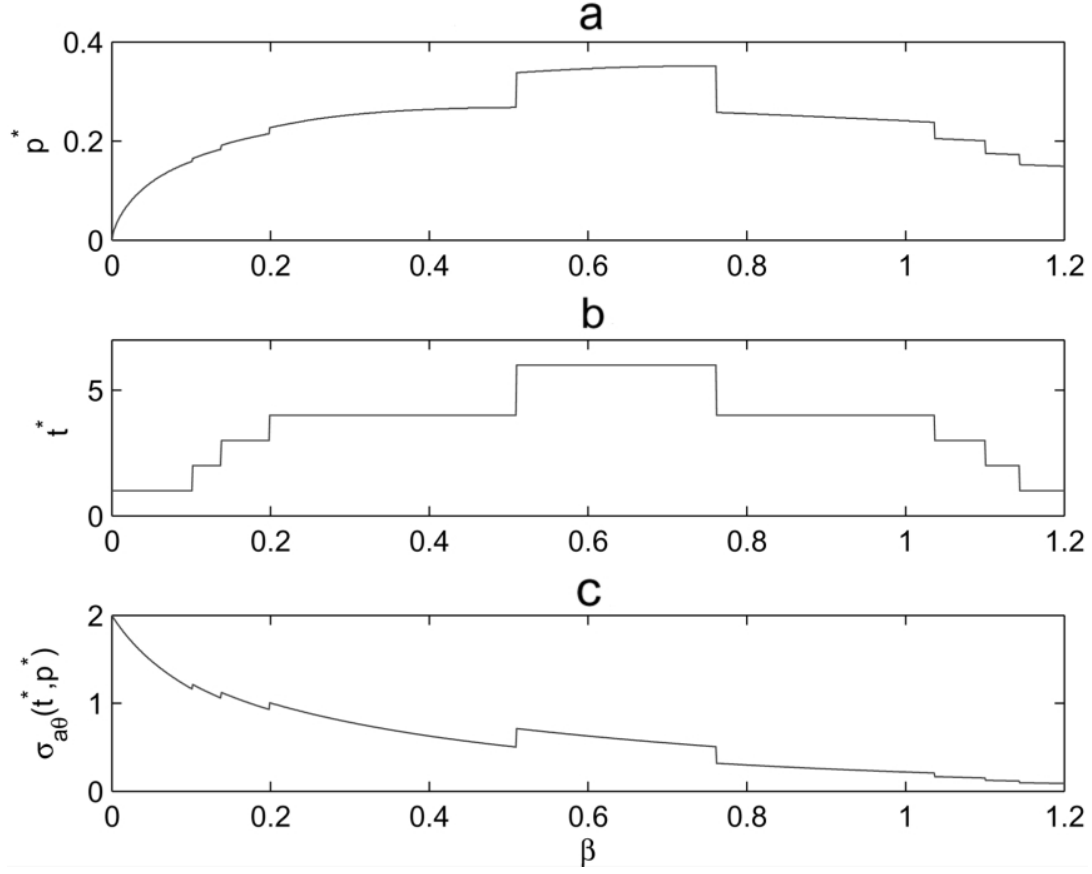

FIG. 4.-Optimal organizational design as a function of the importance of coordination, $\beta$, for the case considered in example 3 (continued): $a$, quality of the horizontal communication, $p^{*} ; b$, task bundling, $t^{*} ; c$, employee flexibility, i.e., $\sigma_{a \theta}\left(t^{*}, p^{*}\right)$.

\section{Task Interdependence, Communication Technology, and Specialization}

Task interdependence and specialization.-We saw in Section IVC that the extent of specialization was nonmonotonic in the degree of task interdependence $\beta$. This result extends when the quality of the communication is an organizational choice variable. Interestingly, also the quality of communication is then nonmonotonic in the degree of task interdependence. The following example illustrates this important result.

EXAMPLE 3 (continued). We consider example 2 again, but now we set $\phi=3$ and investigate the comparative statics with respect to $\beta$ instead. Figures $4 a$ and $b$ illustrate, respectively, how the communication quality and task specialization first increase then decrease as a function of $\beta$. Moreover, $p^{*}$ and $t^{*}$ move up and down together in response to changes in $\beta$. Figure $4 c$ shows how the level of employee flexibility (per unit of $\sigma_{\theta}^{2}$ ) decreases for a given level of $t^{*}$ and $p^{*}$, but it jumps upward (downward) whenever there is an increase (decrease) in task bundling.

Thus, and perhaps counterintuitively, as the importance of tasks being coordinated increases beyond a certain threshold, investments in com- 
munication quality go down rather than up. The reason is that the organization then switches to an ex ante coordination mode with less employee flexibility, diminishing in turn the need for coordination across tasks through task bundling and intensive communication.

Communication technology and specialization.-We close this subsection by investigating the effect of an exogenous change in communication technology on task bundling. As the next example shows, the impact of a drop in the cost of communication, $\delta$, has an impact on task specialization similar to that of an exogenous increase in the communication quality $p$. In particular, when communication costs are high, a drop in $\delta$ results in less specialization, not more. The reason is that the organization then moves from a very rigid organization with limited communication, limited employee flexibility, and extensive specialization to an organization that provides employees with more flexibility and maintains coordination across the different tasks through task bundling and intensive communication.

The main difference with our results in Section IV is that even when communication is perfect, there is a role for task bundling. Indeed, task bundling then serves not only to improve coordination but also to save on communication costs.

EXAMPLE 4. As before, communication is task-to-task with $g(t, p$, $\delta)$ as in example 3 and

$$
h(t, \alpha)=\alpha\left(t+\frac{20}{n-t}\right),
$$

with $\alpha=.25$ and $\phi=3$. The rest of the parameters are as in example 3 . Figure $5 a$ plots $p^{*}$ as a function of $\delta$. Figure $5 b$ shows the degree of task bundling, $t^{*}$, as a function of $\delta$. High values of $\delta$ result in rigid and specialized organizations. As $\delta$ falls below 1.205, the organization is transformed from a very rigid and specialized organization $\left(t^{*}=1\right)$ to an organization with substantial task bundling $\left(t^{*}=6\right)$, intensive horizontal communication $\left(p^{*}=1\right)$, and high employee flexibility $\left(\sigma_{a \theta}=\right.$ $\left.\sigma_{\theta}^{2}\right)$. Thus, for large communication costs, improvements in communication technology and task bundling are complements. Note that even when $p^{*}=1$, there is still a role for task bundling in reducing communication costs. Only when the communication cost parameter $\delta$ falls below .858 , communication has become sufficiently cheap as to warrant more specialization. Thus task bundling and improvements in communication technology are substitutes when communication costs, $\delta$, are sufficiently small. 

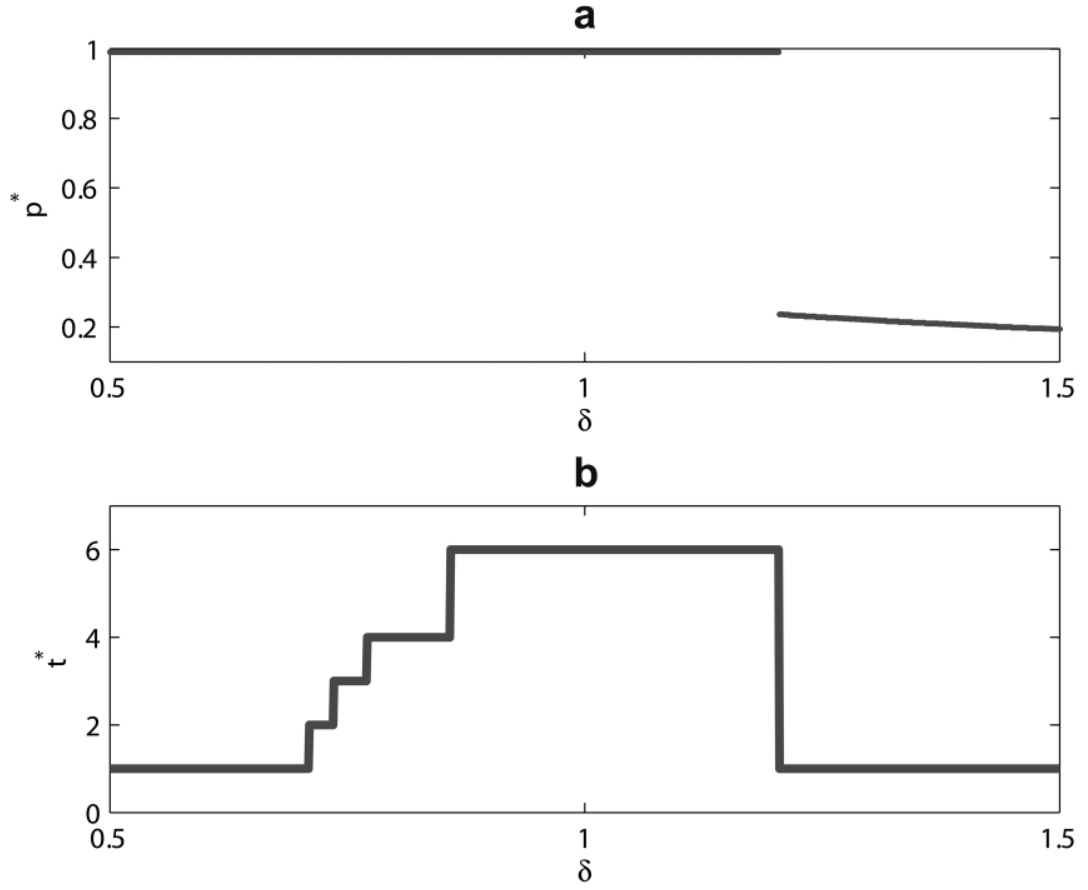

FIG. 5.-Optimal organizational design as a function of the communication cost parameter, $\delta$, for the case considered in example $4: a$, quality of the horizontal communication, $p^{*} ; b$, task bundling, $t^{*}$.

\section{Conclusions}

In this paper we show that the division of labor inside organizations cannot be understood without endogenizing the organization's choice of adaptiveness. In particular, adaptive organizations are information intensive and require task bundling and intensive communication to ensure coordination ex post. In contrast, rigid organizations can rely on rules and task guidelines to coordinate tasks ex ante, allowing such organizations to reap the benefits of specialization.

We consider a model in which adaptation, coordination, and specialization are all endogenously determined and show that this endogeneity induces a profit function for the organization that is often convex in the degree of specialization. In particular, the better the coordination between employees, the more flexibility they receive and hence the higher the returns to further improving coordination. A direct consequence is that organizations tend to be of two types. The first one exploits specialization gains and avoids coordination problems through 
strict work rules, hence sacrificing adaptiveness (ex ante coordination), whereas the second mode provides employees with substantial flexibility to adapt to local circumstances and forgoes the benefits of specialization in order to keep coordination manageable (ex post coordination). As we argue, this is key to rationalizing the trend toward new workplace practices observed in recent decades, which involve broader job definitions and increased employee responsibility.

Our model offers a more subtle picture of some classic results in the literature. First, if there is an increase in the interdependence between tasks and, hence, the importance of coordination, the organization may abandon adaptability altogether, exploit the specialization gains to the fullest, and ensure coordination by limiting the employee's flexibility, that is, by coordinating ex ante. In this case then, more interdependence leads to more specialization, not less. Second, specialization is not monotonic in improvements in communication technology. In the presence of better communication, it pays to be more adaptive, which in turn increases the need for additional coordination. Organizations may meet this additional coordination need by decreasing specialization rather than increasing it, and now improvements in communication are accompanied by broader job descriptions and more employee flexibility.

Throughout the paper we have maintained a team-theoretic framework in which agents share the same organizational goal. While we believe that this is a necessary first step, we are well aware that career concerns and the need to provide individualized incentives may interfere with the objectives of adaptation and coordination. Agents with paternalistic objectives, for example, are unlikely to fully internalize the coordination problems their actions entail for other agents. This may result in overadaptation. Similarly, if communication is privately costly, agents are likely to underinvest in improving coordination. This moral hazard problem may result in suboptimal levels of communication. We leave the study of coordination in the presence of incentives conflicts for future research.

\section{Appendix A}

Technological Trade-offs between Adaptation and Coordination

\section{A. A Model of Technological Trade-offs}

In our model, ${ }^{25}$ coordination problems arise whenever an agent carries out a task in a manner that is not anticipated by other agents, yielding a trade-off between adaptation and coordination. This model is theoretically appealing in that any constraint on coordination is purely informational and hence organi-

${ }^{25}$ We thank one referee and the editor for encouraging us to explore this extension of the model. 
zational in nature. In reality, however, the optimal completion of one task may directly interfere with the completion of other tasks. One way to model such technological task interdependencies is to let each task consist of only a primary action, which now must be adapted to local conditions and coordinated with the primary actions of other tasks. Obviously, this introduces a mechanical tradeoff between adaptation and coordination, which persists even if all tasks are allocated to one agent. In this appendix, we study whether our results are robust to the introduction of such technological trade-offs.

We consider a general model of technological trade-offs. The "one action per task model" described in the previous paragraph and our basic model are two special cases of this general model. In particular, we assume that the organization suffers a quadratic cost $\lambda\left(a^{i j}-a^{i i}\right)^{2}$ whenever a complementary action $a^{i j}$ with $j \neq i$ is set differently from the primary action $a^{i i}$. The cost function associated with task $i$ is thus given by

$$
C^{i}=\phi\left(a^{i i}-\theta^{i}\right)^{2}+\beta \sum_{j \neq i}\left(a^{j i}-a^{i i}\right)^{2}+\lambda \sum_{j \neq i}\left(a^{i j}-a^{i i}\right)^{2}+h(t, \alpha) .
$$

If $\lambda \rightarrow \infty$, primary and complementary actions are then set identical to each other, $a^{i j}=a^{i i}$ for all tasks $i$, and we are in the one action per task model; if $\lambda=0$, there is no relation between primary and complementary actions and we revert to the case studied in the main body of the paper. To focus on the issue of specialization and job design and in the interest of space, we abstract from communication and set $p=0$ throughout and then discuss briefly the implications and difficulties of allowing for communication.

\section{B. Organizational Actions and Performance}

The main difference with our basic model is that the choice of primary action $a^{i i}$ now affects both how well adapted task $i$ is to its environment and how costly it is to coordinate task $i$ with task $j$. This substantially complicates the analysis in that the optimal choice of $a^{i i}$ will depend on the local circumstances of all the tasks $j \in \mathcal{T}(i)$. In contrast, in our basic model, primary actions were tailored to the local information pertaining only to their task. The following proposition characterizes the optimal choice of primary and complementary actions.

Proposition A1. Given a level of task bundling $t$ and faced with a vector of local circumstances $\boldsymbol{\theta}^{\prime}=\left(\theta^{1}, \theta^{2}, \ldots, \theta^{\prime}\right)$, an employee in charge of tasks $i \in$ $\mathcal{T}(i)=\{1, \ldots, t\}$ optimally chooses the following primary actions $a^{i i}$ and complementary actions $a^{i j}$.

$$
a^{i i}(t)=\hat{a}^{i i}+\mu_{\phi}(t)\left(\theta^{i}-\hat{\theta}^{i}\right)+\mu_{\beta}(t) \sum_{j \in \mathcal{T}(i), j \neq i}\left(\theta^{j}-\hat{\theta}^{j}\right)
$$

where $\mu_{\phi}(t)$ and $\mu_{\beta}(t)$ are given in expression (A7) below, and

$$
a^{i j}(t)= \begin{cases}\left(\frac{\beta}{\beta+\lambda}\right) a^{j j}(t)+\left(\frac{\lambda}{\beta+\lambda}\right) a^{i i}(t) & \text { for } j \in \mathcal{T}(i) \\ \left(\frac{\beta}{\beta+\lambda}\right) \hat{a}^{j j}(t)+\left(\frac{\lambda}{\beta+\lambda}\right) a^{i i}(t) & \text { for } j \notin \mathcal{T}(i) .\end{cases}
$$


Proof. The agent in charge of task $i$ minimizes the function

$$
\begin{gathered}
\sum_{l \in \mathcal{T}(i)} \phi\left(a^{l l}-\theta^{l}\right)^{2}+\beta\left\{\sum_{j \in \mathcal{T}(l), j \neq l}\left(a^{j l}-a^{l l}\right)^{2}+\sum_{j \notin \mathcal{T}(l)}\left[\left(\hat{a}^{j l}-a^{l l}\right)^{2}+\left(a^{l j}-\hat{a}^{j j}\right)^{2}\right]\right\} \\
+\lambda \sum_{j \neq l}\left(a^{l j}-a^{l l}\right)^{2}
\end{gathered}
$$

with respect to $\left\{a^{l j}\right\}_{l \in \mathcal{T}(i), j=1,2, \ldots, n}{ }^{26}$ In the expression above, $\hat{a}^{i l}$ for $j \notin \mathcal{T}(l)$ is the average complementary action of the agent in charge of task $j \notin \mathcal{T}(l)$ for task $l .^{27}$ The first-order condition for $a^{j i}(t)$ is immediate and is omitted. As for the first-order condition with respect to $a^{i i}$, and after we demean the variables to eliminate constants, we obtain

$$
a^{i i}(t)=\hat{a}^{i i}+\omega_{\phi}(t)\left(\theta^{i}-\hat{\theta}^{i}\right)+\omega_{\beta}(t) \sum_{j \in \mathcal{T}(i), j \neq i}\left(a^{j j}-\hat{a}^{j j}\right),
$$

where

$$
\begin{aligned}
\omega_{\phi}(t) & =\frac{\phi(\beta+\lambda)}{D(t)}, \\
\omega_{\beta}(t) & =\frac{2 \lambda \beta}{D(t)}, \\
D(t) & =\phi(\beta+\lambda)+\beta^{2}(n-t)+2 \lambda \beta(n-1) .
\end{aligned}
$$

Define $\mathbf{W}_{\beta}(t)$ as the $t \times t$-dimensional matrix with $-\omega_{\beta}(t)$ in its off-diagonal term and 1's in the diagonal terms. Define $\boldsymbol{a}^{p}$ as the $t$-dimensional column vector of the primary actions of the tasks bundled with task $i, \hat{\boldsymbol{a}}^{p}$ as the corresponding $t$ dimensional vector of means (see eq. [A8] below), and $\theta$ the $t$-dimensional column vector of realizations corresponding to those tasks and $\hat{\theta}$ as the corresponding vector of means. Then we can write (A4) in vector notation as

$$
\mathbf{W}_{\beta}(t)\left[\boldsymbol{a}^{p}(t)-\hat{\boldsymbol{a}}^{p}\right]=\omega_{\phi}(t)(\boldsymbol{\theta}-\hat{\boldsymbol{\theta}}) \Rightarrow \boldsymbol{a}^{p}(t)=\hat{\boldsymbol{a}}^{p}+\omega_{\phi}(t) \mathbf{W}_{\beta}^{-1}(t)(\boldsymbol{\theta}-\hat{\boldsymbol{\theta}}),
$$

where the $t \times t$ matrix $\mathbf{W}_{\beta}^{-1}(t)$ has diagonal terms $\varphi_{\phi}(t)$ and off-diagonal terms $\varphi_{\beta}(t)$, with

$$
\begin{aligned}
\varphi_{\phi}(t) & =\frac{1-(t-2) \omega_{\beta}(t)}{1-(t-2) \omega_{\beta}(t)-(t-1) \omega_{\beta}^{2}(t)}, \\
\varphi_{\beta}(t) & =\frac{\omega_{\beta}(t)}{1-(t-2) \omega_{\beta}(t)-(t-1) \omega_{\beta}^{2}(t)} .
\end{aligned}
$$

Finally, define

$$
\mu_{\phi}(t)=\omega_{\phi}(t) \varphi_{\phi}(t), \quad \mu_{\beta}(t)=\omega_{\phi}(t) \varphi_{\beta}(t),
$$

which proves (A2). Finally, the expected primary action, $\hat{a}^{i i}$, is independent of

${ }^{26}$ Notice that the expression features $\hat{a}^{j l}$ and $\hat{a}^{j j}$, which is indeed the optimal forecast since it minimizes the mean square error. Also, when we set $\lambda=0$, the expression collapses to $\sum_{l \in \mathcal{T}(i)} C^{i}$, where $C^{i}$ is now as in expression (4), and we have to set $a^{j l}=a^{u l}$ for $j \in$ $\mathcal{T}(l)$ and $a^{l j}=\hat{a}^{i j}$ for $j \notin \mathcal{T}(l)$ since now there are no technological trade-offs.

${ }^{27}$ Notice that if $l \in \mathcal{T}(i)$, then $\mathcal{T}(i)=\mathcal{T}(l)$. 
$t$, and the $n$-dimensional vector of mean actions is given $b^{28}$

$$
\hat{\boldsymbol{a}}^{p}=\omega_{\phi}(n) \mathbf{W}_{\beta}^{-1}(n) \hat{\boldsymbol{\theta}} .
$$

This completes the proof. QED

As before, we define the level of employee flexibility, $\sigma_{a \theta}(t)$, as the covariance between primary actions and the corresponding local circumstances. It follows immediately from proposition A1 that the level of employee flexibility is given by

$$
\sigma_{a \theta}(t)=\mu_{\phi}(t) \sigma_{\theta}^{2}
$$

It can be shown that when we set $\lambda=0$, that is, when technological trade-offs are absent, the expression for the level of employee flexibility collapses to (7). Moreover, the following proposition shows that, for any $\lambda, \sigma_{a \theta}(t)$ has the same characteristics as in our basic model.

Proposition A2. (a) The level of employee flexibility, $\sigma_{a \theta}(t)$, is increasing in the level of task bundling, that is, $\Delta \sigma_{a \theta}(\bar{t}, \underline{t})>0$ for $\bar{t}, \underline{t} \in \mathcal{F}$; and $(b) \sigma_{a \theta}(n) \leq$ $\sigma_{\theta}^{2}$ with equality if and only if either $\lambda=0$ or $\beta=0$.

Proof. (a) It is enough to show that $\mu_{\phi}(\bar{t})>\mu_{\phi}(\underline{t})$. First notice that $\omega_{\phi}(\bar{t})>$ $\omega_{\phi}(\underline{t})$ and $\omega_{\beta}(\bar{t})>\omega_{\beta}(\underline{t})$ for $\bar{t}>\underline{t} \in \mathcal{J}$. Also,

$$
\begin{aligned}
& \varphi_{\phi}(t)=\frac{1}{1-\frac{(t-1) \omega_{\beta}^{2}(t)}{1-(t-2) \omega_{\beta}(t)}} \\
& \frac{(\bar{t}-1) \omega_{\beta}^{2}(\bar{t})}{1-(\bar{t}-2) \omega_{\beta}(\bar{t})}>\frac{(\underline{t}-1) \omega_{\beta}^{2}(\underline{t})}{1-(\underline{t}-2) \omega_{\beta}(\underline{t})} ;
\end{aligned}
$$

thus $\varphi_{\phi}(\bar{t})>\varphi_{\phi}(\underline{t})$, and so $\mu_{\phi}(\bar{t})>\mu_{\phi}(\underline{t})$. (b) Notice that given the expression in (A5),

$$
\mu_{\phi}(n)=\frac{D(n)-2 \lambda \beta(n-2)}{[D(n)-2 \lambda \beta(n-2)]+2 \lambda \beta(n-1)} \leq 1,
$$

with equality if and only if either $\lambda=0$ or $\beta=0$. QED

Part $a$ of proposition A2 is the key in much of what follows, as it was in the case studied in the main body of the paper: As more tasks are bundled in a single job, employees have more flexibility to adapt to local circumstances. Part $b$ says that now, unlike before, there are limits to the employee flexibility. Even in the presence of complete bundling the level of employee flexibility is lower than the variance of local circumstances, $\sigma_{\theta}^{2}$. The reason, of course, is that the technological trade-offs remain even when the organizational ones are eliminated through complete bundling. We will see below that this affects some results but not others. The next proposition shows, though, that the functional form for the expected profits is identical, up to a constant, to the one in proposition 2.

${ }^{28}$ Here we are slightly abusing notation by using the same symbol for the vectors of means as in expression (A6). In this last expression the means have to be understood as the corresponding $t$-dimensional vector. For instance, for job 1 , the one that bundles the first $t$ tasks, the corresponding vector of mean actions are the first $t$ entries of $\hat{\boldsymbol{a}}^{p}$. 
Proposition A3. The expected profit function is given by

$$
\Pi(t)=-\left(\frac{\beta \lambda}{\beta+\lambda}\right) \sum_{i=1}^{n} \sum_{j \neq i}\left(\hat{a}^{j j}-\hat{a}^{i i}\right)^{2}+n \phi\left[\sigma_{a \theta}(t)-\sigma_{\theta}^{2}\right]-n h(t, \alpha) .
$$

Proof. This follows directly from the substitution of the actions (A2) in the expression for the expected cost function (A1) and the use of the expression for the level of employee flexibility (A9). QED

In (A10) the first term is a constant independent of $t$. We next characterize the optimal choice of task bundling, $t^{*}$, and the trade-off between specialization and adaptability.

\section{The Trade-off between Adaptation and Specialization}

Proposition A4 below shows that, as before, an increase in the environmental uncertainty or a decrease in the returns to specialization results in a decrease in the extent of specialization. Similarly, corollary A1 shows that an increase in the returns to specialization reduces the optimal level of employee flexibility. Corollary Al follows immediately from the proposition and is given without proof.

Proposition A4. Task specialization is decreasing in the variance in local circumstances, $\sigma_{\theta}^{2}$, but increasing in the returns to specialization, $\alpha$.

Proof. Notice that $\Delta \Pi(\bar{t}, \underline{t})=n \phi \Delta \sigma_{a \theta}(\bar{t}, \underline{t})-n \Delta h(\bar{t}, \underline{t})$ for $\bar{t}>\underline{t} \in \mathcal{J}$. Thus $\Delta_{\sigma_{\theta}^{2}} \Pi(\bar{t}, \underline{t})=n \phi \Delta \mu_{\phi}(\bar{t}, \underline{t})>0$, as shown in the proof of proposition A2. Similarly, given assumption (2), $\Delta_{-\alpha} \Pi(\bar{t}, \underline{t})=-\Delta_{-\alpha} h(\bar{t}, \underline{t})>0$. QED

Corollary A1. The level of employee flexibility, $\sigma_{a \theta}\left(t^{*}\right)$, is increasing in the variance of the local circumstances, $\sigma_{\theta}^{2}$, and decreasing in the returns to specialization, $\alpha$.

Interestingly, however, an increase in the importance of being responsive, as measured by $\phi$, to the business environment may have an opposite impact on task specialization. For low values of $\phi$, an increase in the importance of adaptation still results in more task bundling in the one action per task model, and for the same reasons as in our basic model. In contrast, the mechanical trade-off between coordination and adaptation implies that the organization forgoes any attempt to achieve coordination when adaptation becomes very important. Obviously, there is no value to task bundling then, and the organization reverts to full specialization. Intuitively, when concerns for adaptation dwarf coordination needs, tasks are carried out in a stand-alone way, neglecting any externalities with other tasks. This nonmonotonicity of $t^{*}$ in the importance of adaptation is illustrated in the following example.

EXAMPLE A1. The specialization costs are as in example 1 above but with $\alpha=10$. We set $\lambda=\infty$ so that agents are forced to equalize complementary and primary actions, that is, $a^{i j}=a^{i i}$. We set $\beta=1$ and $\sigma_{\theta}^{2}=2$. Finally, $n=24$. Then, numerical computations show that $t^{*}$ is increasing for $\phi \leq 114.15$, when bundling goes from $t^{*}=1$ to $t^{*}=6$, and decreasing for values of $\phi$ higher than that.

\section{The Tenuous Trade-off between Coordination and Specialization}

The trade-off between coordination and specialization is even more tenuous when there is a mechanical trade-off between adaptation and coordination. Intuitively, task bundling is valuable only if employees receive the flexibility to adapt their tasks to local circumstances. But if coordination becomes more im- 
portant, the organization now puts mechanically less emphasis on adaptation. If complete task bundling is expensive, the organization eventually forces all employees to stick to a preagreed course of action, in which case it is also optimal to fully specialize all agents. In particular, if and only if $n h(n, \alpha)>n \phi \sigma_{\theta}^{2}$, then $t^{*}$ is nonmonotone in the importance of coordination $\beta$, with full task specialization being optimal for both very low and very high values of $\beta$. The following example illustrates this result.

ExAmple A1 (continued). The example is as example 4, but now we set $\phi=8$ and $\alpha=1$. As was the case when $\lambda=0$, the extent of specialization is nonmonotone in $\beta$. Indeed $t^{*}$ is increasing when $\beta<1.96$ when bundling goes from $t^{*}=1$ to $t^{*}=8$ and decreasing after that. For $\beta \geq 2.10$, the organization forgoes adaptation altogether and coordinates ex ante, and given this, it is optimal to fully specialize production and set $t^{*}=1$.

\section{E. Communication and Technological Trade-offs}

Extending the model of technological trade-offs to allow for the possibility of communication, whether endogenous or exogenous, is particularly challenging. To illustrate these difficulties, consider the very simple case in which each task consists of one action $(\lambda=\infty)$, the organization consists of only four tasks and two agents $(n=4, t=2)$, and communication is task-to-person. ${ }^{29}$ Let agent $A$ be in charge of $a^{11}$ and $a^{22}$, and agent $B$ in charge of $a^{33}$ and $a^{44}$. Now successful communication of the local information $\theta^{1}$ does not allow us to infer the choice of $a^{11}$ because, as shown in (A2), $a^{11}$ depends on both $\theta^{1}$ and $\theta^{2}$. In addition, $a^{11}$ depends with probability $p(1-p)$ on $\theta^{3}$, with probability $p(1-p)$ on $\theta^{4}$, and with probability $p^{2}$ on both $\theta^{3}$ and $\theta^{4}$. On the other hand, the successful communication of $\theta^{2}$ allows agent $B$ to partially infer the value of $a^{11}$ even when the communication of $\theta^{1}$ is unsuccessful. It follows that primary actions depend on a random number of local information variables since this number of variables is determined by how many communication channels are successful. In contrast, in our basic model, the primary action depends only on the corresponding local information variable. It is this inference problem that makes the case of communication intractable in the model in which technological trade-offs are present. In the example under consideration, for example, for a given realization of $\left(\theta^{1}, \theta^{2}, \theta^{3}, \theta^{4}\right)$, the action vector $\left(a^{11}, a^{22}, a^{33}, a^{44}\right)$ can take 15 different values as opposed to one in our basic model.

\section{Appendix B}

\section{Proofs}

\section{Proof of Proposition 1}

Clearly the choice of $a^{j i}$ for $j \in \mathcal{T}(i), j \neq i$, or $j \notin \mathcal{T}(i)$ when the agent in charge of task $j$ observes $a^{i i}$ is $a^{j i}=a^{i i}$. When instead the agent in charge of task $j \notin$ $T(i)$ does not observe $a^{i i}$, the agent minimizes $E\left[\beta\left(a^{j i}-a^{i i}\right)^{2}\right]$ and the solution is $a^{j i}=E\left(a^{i i}\right)$. The choice of $a^{i i}$ then follows from the minimization of the

${ }^{29}$ Task-to-person communication implies that the first agent needs to communicate $\theta^{1}$ only once to the second agent. In total, there are thus four communication channels, one for each task. 
function,

$$
\min _{a^{i i}}\left\{\phi\left(a^{i i}-\theta^{i}\right)^{2}+\beta(1-p)(n-t)\left[E\left(a^{i i}\right)-a^{i i}\right]^{2}\right\},
$$

and the solution is given by

$$
a^{i i}=\left[\frac{\phi}{\phi+B(t)}\right] \theta^{i}+\left[\frac{B(t)}{\phi+B(t)}\right] E\left(a^{i i}\right),
$$

where

$$
B(t)=\beta(n-t)(1-p) .
$$

Clearly then $E\left(a^{i i}\right)=\hat{\theta}^{i}$. QED

Proof of Proposition 2

Substitution of (B2) in (B1) together with the fact that $a^{j i}=E\left(a^{i i}\right)=\hat{\theta}^{i}$ for $j \notin \mathcal{T}(i)$ when the agent in charge of task $j$ does not observe $a^{i i}$ yields

$$
\phi\left[\frac{B(t)}{\phi+B(t)}\right]\left(\theta^{i}-\hat{\theta}^{i}\right)^{2} .
$$

Given that there are $n$ tasks, the expected adaptation and coordination costs are

$$
n \phi\left[\frac{B(t)}{\phi+B(t)}\right] \sigma_{\theta}^{2}=n \phi\left[\sigma_{\theta}^{2}-\sigma_{a \theta}(t)\right] .
$$

Adding $n h(t, \alpha)$ yields $-\Pi(t)$. QED

\section{Proof of Proposition 3}

Preliminaries.-We apply standard concepts in the mathematics of complementarities to prove this result. Briefly we show that the function $\Pi(t, \tau)$ is supermodular in $t$ and $\tau$, where $\tau \in\left\{\phi, \sigma_{\theta}^{2},-\alpha\right\}$. For this it is enough to check that the "cross-derivatives" are positive. In our case, the number of tasks $t$ assigned to a job is a discrete variable in $\mathcal{J}=\{t \in \mathbb{N}$ such that $(n / t) \in \mathbb{N}\}$, and thus we have to show that $\Delta_{\tau} \Pi(\bar{t}, \underline{t}) \geq 0$ for $\bar{t}>\underline{t} \in \mathcal{J}$ (see expression [1]), which simply says that the returns to increasing task bundling from $\underline{t}$ to $\bar{t}$ increase with $\tau$. Supermodularity of $\Pi(t, \tau)$ is enough to yield the desired comparative statics of the choice variable $t$ with respect to $\tau \cdot{ }^{30}$ Also notice that for our purposes it is enough to show that the function is supermodular in $t$ and each of the parameters; there is no need to control for the interactions between the parameters.

Proof of proposition 3.-Monotonicity of $t^{*}$ with respect to $\phi$ : Trivial algebra

${ }^{30}$ See Milgrom and Roberts (1990, sec. I) for a clear and precise summary of these tools. For a less technical primer, see Milgrom and Roberts (1995). 
shows that

$$
\Delta_{\phi} \Pi(\bar{t}, \underline{t})=\left\{\left[\frac{B(\underline{t})}{\phi+B(\underline{t})}\right]^{2}-\left[\frac{B(\bar{t})}{\phi+B(\bar{t})}\right]^{2}\right\} \sigma_{\theta}^{2},
$$

where $B(t)$ was defined in equation (B3). Clearly $B(\underline{t})>B(\bar{t})$, and thus $\Delta_{\phi} \Pi(\bar{t}$, t) $\geq 0$.

Monotonicity with respect to $\sigma_{\theta}^{2}$ : Trivially, $\Delta_{\sigma_{\theta}^{2}} \Pi(\bar{t}, \underline{t})=\sigma_{\theta}^{-2} n \phi \Delta \sigma_{a \theta}(\bar{t}, \underline{t})>0$, given (8)

Monotonicity with respect to $-\alpha$ : Clearly $\Delta_{-\alpha} \Pi(\bar{t}, \underline{t})=\Delta_{\alpha} h(\bar{t}, \underline{t})>0$ given (2). QED

\section{Proof of Proposition 4}

Part $a$ : As $\beta \rightarrow \infty$, any coordination failure will be infinitely costly, and thus the organization will find it necessary to set $a^{j i}=a^{i i}$ for all $i, j$ at the optimum. Given that $p<1, t^{*}$ converges to either $t^{*}=1$ or $t^{*}=n$. Indeed assume that instead $1<t^{*}<n$. Then because $\beta \rightarrow \infty$, it is optimal to set $a^{j i}=a^{i i}=\hat{\theta}^{i i}$ for all $j$ and $i$. However, in this case, because there are positive returns to specialization, it pays for the organization to set $t^{*}=1$. Alternatively $t^{*}=n$, in which case the trade-off between coordination and adaptation is absent. Clearly, if $\phi=0$, $t^{*}=1$ will be optimal. Assume next that, in addition to $\beta, \phi$ also tends to infinity. In this case any deviation from perfect coordination or perfect adaptation results in profits also going to minus infinity. Because $h(t, \alpha)<\infty$, it is optimal to set $t^{*}=n$, which guarantees perfect coordination and adaptation at the expense of (finite) specialization costs. From proposition 3, $t$ is monotone in $\phi$. Thus if $t^{*}=n$ for some $\hat{\phi}$, then also $t^{*}=n$ for $\phi \geq \hat{\phi}$. Clearly, $\hat{\phi}$ is the adaptation parameter for which the organization is indifferent between full specialization of full bundling, that is,

$$
-h(n, \alpha)=-\hat{\phi} \sigma_{\theta}^{2}-h(1, \alpha) \Rightarrow \hat{\phi}=\frac{\Delta h(n, 1)}{\sigma_{\theta}^{2}},
$$

and thus by (2), $\hat{\phi}$ is increasing in $\alpha$.

Part $b$ : Given part $a$ and $\phi<\hat{\phi}$, we know that $t$ must be decreasing in $\beta$ for some values of $\beta>\hat{\beta}$. We thus need to show only that $t$ is weakly decreasing for any value of $\beta>\hat{\beta}$. Assume not and define $t^{*}(\hat{\beta})=\hat{t}$. Then there must exist a $\left\{\beta_{1}, \beta_{2}\right\}$ and $\left\{t_{1}, t_{2}\right\}$ with $\beta_{2}>\beta_{1}>\hat{\beta}$ and $\hat{t}>t_{2}>t_{1}$ such that $\Delta \Pi\left(\hat{t}, t_{1}, \beta_{1}\right)=0$, $\Delta \Pi\left(t_{2}, t_{1}, \beta_{2}\right)=0, \Delta_{\beta} \Pi\left(\hat{t}, t_{1}, \beta_{1}\right) \leq 0$, and $\Delta_{\beta} \Pi\left(t_{2}, t_{1}, \beta_{2}\right) \geq 0$. From (10) and $\beta_{2}>\beta_{1}, \Delta_{\beta} \Pi\left(\hat{t}, t_{1}, \beta_{1}\right) \leq 0$ implies that $\Delta_{\beta} \Pi\left(\hat{t}, t_{1}, \beta_{2}\right)<0$. But from (10) and $\hat{t}>$ $t_{2}$, also $\Delta_{\beta} \Pi\left(t_{2}, t_{1}, \beta_{2}\right)<0$, a contradiction. QED

\section{Proof of Proposition 5}

Part $a$ : Since $t^{*}=1$, if $\phi=0$ and $t^{*}$ is increasing in $\phi$ (proposition 3), it follows that there exists a $\phi^{\prime}$ such that $t^{*}=1$ if and only if $\phi \leq \phi^{\prime}$. Moreover, since $t^{*}$ is increasing in $\alpha$ (proposition 3), $\phi^{\prime}$ is increasing in $\alpha$.

Part $b$ : Given part $a$ and $\phi<\phi^{\prime}$, we know that $t$ must be increasing in $p$ for some values of $p<\hat{p}$. We thus need to show only that $t$ is weakly increasing for 
any value of $p<\hat{p}$. Assume not and define $t^{*}(\hat{p})=\hat{t}$. Then there exists a $\left\{p_{1}\right.$, $\left.p_{2}\right\}$ and $\left\{t_{1}, t_{2}\right\}$ with $p_{1}<p_{2}<\hat{p}$ and $\hat{t}>t_{1}>t_{2}$ such that $\Delta \Pi\left(t_{1}, t_{2}, p_{1}\right)=0, \Delta \Pi(\hat{t}$, $\left.t_{2}, p_{2}\right)=0, \Delta_{p} \Pi\left(t_{1}, t_{2}, p_{1}\right) \leq 0$, and $\Delta \Pi_{p}\left(\hat{t}, t_{2}, p_{2}\right) \geq 0$. From (11) and $p_{2}>p_{1}$, $\Delta_{p} \Pi\left(t_{1}, t_{2}, p_{1}\right) \leq 0$ implies that $\Delta_{p} \Pi\left(t_{1}, t_{2}, p_{2}\right)<0$. But from (10) and $\hat{t}>t_{1}$, also $\Delta_{p} \Pi\left(\hat{t}, t_{2}, p_{2}\right)<0$, a contradiction. QED

\section{Proof of Proposition 6}

Preliminaries.-As discussed in Section $\mathrm{V} B$, in order to establish the desired comparative statics, it is convenient to work with expression (14), reproduced next for convenience:

$$
\pi(t, \hat{p})=\max _{p \geq \hat{p}} \Pi(t, p)
$$

The choice of $\hat{p}$ then does not affect the optimal choice of the other design variables since, given the strict quasi concavity of the profit function, the highest optimal value of $\hat{p}$ is always chosen to be equal to $p^{*}(t)$, the unique optimized value for the quality of communication. With some abuse of notation, define $\tilde{p}(t)$ as the value of the constrained maximization of the profit function:

$$
\tilde{p}(t)= \begin{cases}p^{*}(t) & \text { if } p^{*}(t)>\hat{p} \\ \hat{p} & \text { if } p^{*}(t) \leq \hat{p}\end{cases}
$$

Recall as well that we have assumed that $\Pi(t, p)$ is a strictly quasi-concave function of $p$ so that it is also a differentiable function of $p$. As a consequence, it follows that $p^{*}(t)$ is the solution of $\Pi_{p}(t, p)=0$, where

$$
\Pi_{p}(t, p)=-n\left[\frac{\phi}{\phi+B(t, p)}\right]^{2} B_{p}(t) \sigma_{\theta}^{2}-g_{p}(t, p, \delta),
$$

with $B_{p}(t)=-\beta(n-t)$ and $B(t, p)$ is defined in expression (B3) with the corresponding change in notation to account for the endogeneity of $p$. In addition, recall that we have assumed that $g_{p}(t, 0, \delta)=0$ and $\lim _{p \rightarrow 1} g_{p}(t, p, \delta)=+\infty$; it follows that $p^{*}(t)$ is unique, strictly in the interior of $[0,1]$, and differentiable with respect to any of the parameters $\tau \in\left\{\sigma_{\theta}^{2}, \phi,-\alpha\right\}$. Notice that

$$
B_{\hat{p}}(t)= \begin{cases}0 & \text { if } p^{*}(t)>\hat{p} \\ -\beta(n-t) & \text { if } p^{*}(t) \leq \hat{p}\end{cases}
$$

The next lemma is instrumental in the proof of proposition 6 below. Lemma A1. $\tilde{p}(\bar{t})>\tilde{p}(\underline{t})$ for $\bar{t}>\underline{t} \in \mathcal{J}$. 
Proof. It is enough to show that $p^{*}(\bar{t})>p^{*}(\underline{t})$. Notice that given assumption 1 ,

$$
\begin{aligned}
0 & =\Pi_{p}\left(\underline{t}, p^{*}(\underline{t})\right) \\
& =(n-\underline{t})\left(\frac{n \beta}{\left\{\phi+\beta(n-\underline{t})\left[1-p^{*}(\underline{t})\right]\right\}^{2}} \sigma_{\theta}^{2}-\frac{g_{p}\left(\underline{t}, p^{*}(\underline{t})\right)}{n-\underline{t}}\right) \\
& <(n-\underline{t})\left(\frac{n \beta}{\left\{\phi+\beta(n-\underline{t})\left[1-p^{*}(\underline{t})\right]\right\}^{2}} \sigma_{\theta}^{2}-\frac{g_{p}\left(\bar{t}, p^{*}(\underline{t})\right)}{n-\bar{t}}\right) \\
& =\left(\frac{n-\underline{t}}{n-\bar{t}}\right)\left(\frac{n \beta(n-\bar{t})}{\left\{\phi+\beta(n-\underline{t})\left[1-p^{*}(\underline{t})\right]\right\}^{2}} \sigma_{\theta}^{2}-g_{p}\left(\bar{t}, p^{*}(\underline{t})\right)\right) \\
& <\left(\frac{n-\underline{t}}{n-\bar{t}}\right)\left(\frac{n \beta(n-\bar{t})}{\left\{\phi+\beta(n-\bar{t})\left[1-p^{*}(\underline{t})\right]\right\}^{2}} \sigma_{\theta}^{2}-g_{p}\left(\bar{t}, p^{*}(\underline{t})\right)\right) \\
& =\left(\frac{n-\underline{t}}{n-\bar{t}}\right) \Pi_{p}\left(\bar{t}, p^{*}(\underline{t})\right),
\end{aligned}
$$

and $\Pi_{p}\left(\bar{t}, p^{*}(\underline{t})\right)>0$. Thus by assumption 2 , the strict quasi concavity of $\Pi(t, p)$, $p^{*}(\bar{t})>p^{*}(\underline{t})$. QED

As in proposition 3 , our strategy to prove proposition 6 is to show that $\pi(t$, $\hat{p}, \tau)$, with $\tau \in\left\{\phi, \sigma_{\theta}^{2},-\alpha\right\}$, is supermodular in $t, \hat{p}$, and $\tau$, and thus $t^{*}$ and $p^{*}$ are monotone in $\tau$.

Proof of proposition 6.-First we show that $\Delta_{\hat{p}} \Pi(\bar{t}, \underline{t}) \geq 0$. Use lemma A1 and assume first that $p^{*}(\bar{t})>p^{*}(\underline{t})>\hat{p}$. Then clearly $\Delta_{\hat{p}} \Pi(\bar{t}, \underline{t})=0$. Next, if $p^{*}(\bar{t})>$ $\hat{p}>p^{*}(t)$, then $\Delta_{\hat{p}} \Pi(\bar{t}, \underline{t})=-\pi_{\hat{p}}(t, \hat{p})>0$, by the strict quasi concavity of the profit function with respect to $p$. Assume finally that $\hat{p}>p^{*}(\bar{t})>p^{*}(\underline{t})$. Then

$$
\Delta_{\hat{p}} \Pi(\bar{t}, \underline{t})=n \phi \Delta_{\hat{p}} \sigma_{a \theta}(\bar{t}, \underline{t})-\Delta_{\hat{p}} g(\bar{t}, \underline{t}) .
$$

First, notice that given assumption 1,

$$
\begin{aligned}
-\Delta_{\hat{p}} g(\bar{t}, \underline{t}) & =n(n-\underline{t})\left[\frac{g_{\hat{p}}(\underline{t}, \hat{p})}{n(n-\underline{t})}-\left(\frac{n-\bar{t}}{n-\underline{t}}\right) \frac{g_{\hat{p}}(\bar{t}, \hat{p})}{n(n-\bar{t})}\right] \\
& >(n-\underline{t}) \frac{g_{\hat{p}}(\bar{t}, \hat{p})}{n-\bar{t}}\left(1-\frac{n-\bar{t}}{n-\underline{t}}\right) \\
& =\left(\frac{\bar{t}-\underline{t}}{n-\bar{t}}\right) g_{\hat{p}}(\bar{t}, \hat{p}) .
\end{aligned}
$$


Next, notice that

$$
\begin{aligned}
n \phi \Delta_{\hat{p}} \sigma_{a \theta}(\bar{t}, \underline{t}) & =n \phi^{2} \sigma_{\theta}^{2}\left\{\frac{\beta(n-\bar{t})}{[\phi+\beta(n-\bar{t})(1-\hat{p})]^{2}}-\frac{\beta(n-\underline{t})}{[\phi+\beta(n-\underline{t})(1-\hat{p})]^{2}}\right\} \\
& >n \phi^{2} \sigma_{\theta}^{2}\left\{\frac{\beta(n-\bar{t})}{[\phi+\beta(n-\bar{t})(1-\hat{p})]^{2}}-\frac{\beta(n-\underline{t})}{[\phi+\beta(n-\bar{t})(1-\hat{p})]^{2}}\right\} \\
& =-n \phi^{2} \sigma_{\theta}^{2} \frac{\beta(\bar{t}-\underline{t})}{[\phi+\beta(n-\bar{t})(1-\hat{p})]^{2}} \\
& =-\left(\frac{\bar{t}-\underline{t}}{n-\bar{t}}\right)\left[\frac{n \phi^{2} \beta(n-\bar{t})}{\phi+\beta(n-\bar{t})(1-\hat{p})}\right] .
\end{aligned}
$$

Thus

$$
\begin{aligned}
\Delta_{\hat{p}} \Pi(\bar{t}, \underline{t}) & >-\left(\frac{\bar{t}-\underline{t}}{n-\bar{t}}\right)\left[\frac{n \phi^{2} \beta(n-\bar{t})}{\phi+\beta(n-\bar{t})(1-\hat{p})}-g_{\hat{p}}(\bar{t}, \hat{p})\right] \\
& =-\left(\frac{\bar{t}-\underline{t}}{n-\bar{t}}\right) \pi_{\hat{p}}(\bar{t}, \hat{p}) .
\end{aligned}
$$

But $\pi_{\hat{p}}(\bar{t}, \hat{p})<0$ by the fact that $\hat{p}>p(\tilde{t})$ and the strict quasi concavity of the profit function with respect to $p$.

Finally, to prove the monotonicity of $t^{*}$ and $p^{*}$ with respect to the parameters of interest, we proceed as in proposition 3. The proof of the complementarity of the design variables with $\sigma_{\theta}^{2}$ and $-\alpha$ is immediate and is omitted in the interest of space. The proof of the complementarity between $t$ and $\phi$ is identical to that of proposition 3 . As for the complementarity of $\hat{p}$ with respect to $\phi$, notice that

$$
\pi_{\hat{p}}(t, \hat{p})=n \beta(n-t)\left[\frac{\phi}{\phi+\beta(n-t)(1-\hat{p})}\right]^{2} \sigma_{\theta}^{2}-g_{\hat{p}}(t, \hat{p})
$$

and

$$
\pi_{\hat{p} \phi}(t, \hat{p})=2 n \beta(n-t)\left\{\frac{\phi \beta(n-t)(1-\hat{p})}{[\phi+\beta(n-t)(1-\hat{p})]^{2}}\right\} \sigma_{\theta}^{2}>0,
$$

which concludes the proof. QED

\section{References}

Aoki, Masahiko. 1986. "Horizontal vs. Vertical Information Structure of the Firm.” A.E.R. 76 (December): 971-83.

Argote, Linda. 1982. "Input Uncertainty and Organizational Coordination in Hospital Emergency Units." Administrative Sci. Q. 27 (September): 420-34.

Becker, Gary S., and Kevin M. Murphy. 1992. "The Division of Labor, Coordination Costs, and Knowledge." O.J.E. 107 (November): 1137-60.

Bolton, Patrick, and Mathias Dewatripont. 1994. "The Firm as a Communication Network.” Q.J.E. 109 (November): 809-39.

Bresnahan, Timothy F., Erik Brynjolfsson, and Lorin M. Hitt. 2002. "Information 
Technology, Workplace Organization, and the Demand for Skilled Labor: Firm-Level Evidence." Q.J.E. 117 (February): 339-76.

Brynjolfsson, Erik, and Lorin M. Hitt. 1998. "Information Technology and Organizational Design: Evidence from Micro Data." Manuscript, Massachusetts Inst. Tech.

Caroli, Eve. 2001. "New Technologies, Organizational Change, and the Skill Bias: What Do We Know?" In Technology and the Future of European Employment, edited by Pascal Petit and Luc Soete. London: Elgar.

Caroli, Eve, and John Van Reenen. 2001. "Skill-Biased Organizational Change? Evidence from a Panel of British and French Establishments." Q.J.E. 116 (November): 1449-92.

Coutrot, Thomas. 1996. "Les noveaux modes d'organisation de la production: Quels effets sur l'emploi, la formation, l'organisation du travail?” In Donnees sociales: Le société française. Paris: INSEE.

Cremer, Jacques. 1980. "A Partial Theory of the Optimal Organization of a Bureaucracy." Bell J. Econ. 11 (Autumn): 683-93.

Crowston, Kevin. 1997. "A Coordination Theory Approach to Organizational Process Design.” Organization Sci. 8 (March/April): 157-75.

Curtis, Bill, Herb Krasner, and Neil Iscoe. 1988. "A Field Study of the Software Design Process for Large Systems.” Communications ACM 31 (November): $1268-87$.

Dessein, Wouter, and Tano Santos. 2003. "The Demand for Coordination.” Working Paper no. 10056 (November), NBER, Cambridge, MA.

Faraj, Samer, and Lee Sproull. 2000. "Coordinating Expertise in Software Development Teams." Management Sci. 46 (December): 1554-68.

Garicano, Luis. 2000. "Hierarchies and the Organization of Knowledge in Production." J.P.E. 108 (October): 874-904.

Geanakoplos, John, and Paul Milgrom. 1991. "A Theory of Hierarchies Based on Limited Managerial Attention.” J. Japanese and Internat. Econ. 5 (September): 205-25.

Hammer, Michael, and James Champy. 2001. Reengineering the Corporation: A Manifesto for Business Revolution. 2nd ed. New York: HarperBus.

Holmstrom, Bengt, and Paul Milgrom. 1994. "The Firm as an Incentive System." A.E.R. 84 (September): 972-91.

Ichniowski, Casey, Thomas A. Kochan, David Levine, Craig Olson, and George Strauss. 1996. "What Works at Work: Overview and Assessment." Indus. Relations 35 (July): 299-333.

Ichniowski, Casey, Kathryn Shaw, and Giovanna Prennushi. 1995. "The Effects of Human Resource Management Practices on Productivity.” Working Paper no. 5333 (November), NBER, Cambridge, MA.

. 1997. "The Effects of Human Resource Management Practices on Productivity: A Study of Steel Finishing Lines.” A.E.R. 87 (June): 291-313.

Kraut, Robert E., and Lynn A. Streeter. 1995. "Coordination in Software Development." Communications ACM 38 (March): 69-81.

Lincoln, James R., Mitsuyo Hanada, and Kerry McBride. 1986. "Organizational Structures in Japanese and US Manufacturing." Administrative Sci. Q. 31 (September): 338-64.

Lindbeck, Assar, and Dennis J. Snower. 2000. "Multitask Learning and the Reorganization of Work: From Tayloristic to Holistic Organization." J. Labor Econ. 18 (July): 353-76.

March, James G., and Herbert A. Simon. 1958. Organizations. New York: Wiley. 
Marschak, Jacob, and Roy Radner. 1972. Economic Theory of Teams. New Haven, CT: Yale Univ. Press.

Milgrom, Paul, and John Roberts. 1990. "The Economics of Modern Manufacturing: Technology, Strategy, and Organization." A.E.R. 80 (June): 511-28.

1995. "Complementarities and Fit: Strategy, Structure, and Organizational Change in Manufacturing." J. Accounting and Econ. 19 (April): 179-208.

OECD. 1999. Employment Outlook. Paris: OECD.

Osterman, Paul. 1994. "How Common Is Workplace Transformation and Who Adopts It?" Indus. and Labor Relations Rev. 47 (January): 173-88.

Radner, Roy. 1993. "The Organization of Decentralized Information Processing." Econometrica 61 (September): 1109-46.

Rivkin, Jan W., and Nicolaj Siggelkow. 2003. "Balancing Search and Stability: Interdependencies among Elements of Organizational Design.” Management Sci. 49 (March): 290-311.

Smith, Adam. [1776] 1981. An Inquiry into the Nature and Causes of the Wealth of Nations. Indianapolis: Liberty Classics.

Van de Ven, Andrew H., Andre L. Delbecq, and Richard Koenig Jr. 1976. "Determinants of Coordination Modes within Organizations." American Sociological Rev. 41 (April): 322-38.

Van Zandt, Timothy. 1999. "Real-Time Decentralized Information Processing as a Model of Organizations with Boundly Rational Agents." Rev. Econ. Studies 66 (July): 633-58.

Vayanos, Dimitri. 2003. "The Decentralization of Information Processing in the Presence of Interactions." Rev. Econ. Studies 70 (July): 667-95.

Zmud, Robert W. 1980. "Management of Large Software Development Efforts." MIS Q. 4 (June): 45-55. 